\title{
A Two-Scale Discretization Scheme for Mixed Variational Formulation of Eigenvalue Problems
}

\author{
Yidu Yang, ${ }^{1}$ Wei Jiang, ${ }^{2}$ Yu Zhang, ${ }^{1}$ Wenjun Wang, ${ }^{1}$ and Hai Bi ${ }^{1}$ \\ ${ }^{1}$ School of Mathematics and Computer Science, Guizhou Normal University, Guiyang 550001, China \\ ${ }^{2}$ School of Physics and Mechanical \& Electrical Engineering, Xiamen University, Xiamen 361005, China \\ Correspondence should be addressed to Yidu Yang, ydyang@gznu.edu.cn
}

Received 1 March 2012; Accepted 24 April 2012

Academic Editor: Ibrahim Sadek

Copyright (c) 2012 Yidu Yang et al. This is an open access article distributed under the Creative Commons Attribution License, which permits unrestricted use, distribution, and reproduction in any medium, provided the original work is properly cited.

This paper discusses highly efficient discretization schemes for mixed variational formulation of eigenvalue problems. A new finite element two-scale discretization scheme is proposed by combining the mixed finite element method with the shifted-inverse power method for solving matrix eigenvalue problems. With this scheme, the solution of an eigenvalue problem on a fine grid $K^{h}$ is reduced to the solution of an eigenvalue problem on a much coarser grid $K^{H}$ and the solution of a linear algebraic system on the fine grid $K^{h}$. Theoretical analysis shows that the scheme has high efficiency. For instance, when using the Mini element to solve Stokes eigenvalue problem, the resulting solution can maintain an asymptotically optimal accuracy by taking $H=O(\sqrt[4]{h})$, and when using the $P_{k+1}-P_{k}$ element to solve eigenvalue problems of electric field, the calculation results can maintain an asymptotically optimal accuracy by taking $H=O(\sqrt[3]{h})$. Finally, numerical experiments are presented to support the theoretical analysis.

\section{Introduction}

To improve the efficiency of finite element method, $\mathrm{Xu}$ introduced a two-scale discretization scheme and applied it to nonsymmetric and nonlinear elliptic problems (see [1-3]). Later on, this scheme attracted the attention of academic circles and has been successfully applied to Stokes equations (see [4-7]), semilinear eigenvalue problems (see [8]), and linear differential operator eigenvalue problems, and so forth.

Up to now, two kinds of finite element two-scale discretization schemes have been developed for linear differential operator eigenvalue problems. The first kind is established by Xu and Zhou [9] in 2001, whose idea correlates with the iterative Galerkin method which was established by Lin and Xie [10] and Sloan [11], but it bases on the finite element spaces on two different scale grids. The scheme of $\mathrm{Xu}$ and Zhou has been applied to 
the conforming finite element method for electric structure eigenvalue problem (see [12$14]$ ), the conforming/nonconforming finite element method for non-self-adjoint eigenvalue problem (see $[15,16])$, the conforming/nonconforming finite element method for Steklov eigenvalue problem (see $[17,18]$ ), the mixed finite element method for Stokes eigenvalue problem, and biharmonic equations eigenvalue problem (see $[19,20])$. Another group of two-scale discretization scheme is proposed by Yang and Bi [21]. They established the conforming/nonconforming finite element two-scale discretization scheme based on the shifted-inverse power method.

With two-scale discretization schemes, the solution of an eigenvalue problem on a fine grid $K^{h}$ is reduced to the solution of an eigenvalue problem on a much coarser grid $K^{H}$ and the solution of a linear algebraic system on the fine grid $K^{h}$, and the resulting solution still maintains an asymptotically optimal accuracy. Thus, the computational efficiency is improved.

Influenced by the work mentioned above, in this paper we establish a new finite element two-scale discretization scheme for mixed variational formulation of eigenvalue problem and apply it to Stokes eigenvalue problem and eigenvalue problem of electric field. The research of this paper has the following features.

(1) Our two-scale discretization scheme is a combinative production of the mixed finite element method and the shifted-inverse power method (see [22, Algorithm 27.2]). Comparing with the scheme in $[19,20]$, the scheme in this paper is more efficient: the resulting solution obtained by our scheme can maintain an asymptotically optimal accuracy by taking $H=O(\sqrt[4]{h})$ when solving Stokes eigenvalue problem and $H=O(\sqrt[3]{h})$ when solving eigenvalue problem of electric field; however, with the scheme in $[19,20]$ the resulted solution maintains an asymptotically optimal accuracy by taking $H=O(\sqrt[2]{h})$.

(2) The literatures of high-efficient numerical method for eigenvalue problem of electric field are not too many by now, thus they seem to be very valuable. Our two-scale discretization scheme is a new and highly efficient method for eigenvalue problem of electric field.

The rest of this paper is organized as follows. Some preliminaries of finite element approximations for eigenvalue problems which are needed in this paper are provided in the next section. In Section 3, for eigenvalue problem mixed variational formulation (2.3)(2.4) in general form, the finite element two-scale discretization scheme based on the shiftedinverse power method is established, and the validity of this scheme is proved theoretically. In Sections 4 and 5, the scheme established in Section 3 is applied to Stokes eigenvalue problem and eigenvalue problem of electric field, respectively. Finally, numerical experiments are presented in Section 6.

\section{Preliminaries}

Let $V, W$, and $D$ be three real Hilbert spaces with inner products and norms $(\cdot, \cdot)_{V},\|\cdot\|_{V},(\cdot, \cdot)_{W}$, $\|\cdot\|_{W}$, and $(\cdot, \cdot)_{D},\|\cdot\|_{D}$, respectively. We suppose that $V \hookrightarrow D$ (continuously imbedded), $a(\cdot, \cdot)$ is a symmetric, continuous, and $V$-elliptic bilinear form on $V \times V$, that is,

$$
\begin{gathered}
|a(q, \psi)| \leq M_{1}\|q\|_{V}\|\psi\|_{V^{\prime}} \quad \forall q, \psi \in V, \\
a(q, q) \geq v\|q\|_{V^{\prime}}^{2} \quad \forall 0 \neq q \in V,
\end{gathered}
$$


$b(\cdot, \cdot)$ is a continuous bilinear form on $V \times W$, that is,

$$
|b(\psi, v)| \leq M_{2}\|\psi\|_{V}\|v\|_{W}, \quad \forall \psi \in V, v \in W
$$

In scientific and engineering computations, many eigenvalue problems for differential equation have the following mixed variational formulation.

Find $(\lambda, u, \sigma) \in \mathbf{R} \times V \times W,(u, \sigma) \neq(0,0)$, such that

$$
\begin{gathered}
a(u, \psi)+b(\psi, \sigma)=\lambda(u, \psi)_{D^{\prime}}, \quad \forall \psi \in V, \\
b(u, v)=0, \quad \forall v \in W .
\end{gathered}
$$

In order to solve problem (2.3)-(2.4), one should construct finite element spaces $V_{h} \subset V$ and $W_{h} \subset W$. Restricting (2.3)-(2.4) on $V_{h} \times W_{h}$ we get the conforming mixed finite element approximation as follows. Find $\left(\lambda_{h}, u_{h}, \sigma_{h}\right) \in \mathbf{R} \times V_{h} \times W_{h},\left(u_{h}, \sigma_{h}\right) \neq(0,0)$, such that

$$
\begin{gathered}
a\left(u_{h}, \psi\right)+b\left(\psi, \sigma_{h}\right)=\lambda_{h}\left(u_{h}, \psi\right)_{D^{\prime}} \quad \forall \psi \in V_{h}, \\
b\left(u_{h}, v\right)=0, \quad \forall v \in W_{h} .
\end{gathered}
$$

Consider the associated source and approximate source problems.

Given $f \in D$, find $(w, p) \in V \times W$ satisfying

$$
\begin{gathered}
a(w, \psi)+b(\psi, p)=(f, \psi)_{D^{\prime}}, \quad \forall \psi \in V, \\
b(w, v)=0, \quad \forall v \in W .
\end{gathered}
$$

Given $f \in D$, find $\left(w_{h}, p_{h}\right) \in V_{h} \times W_{h}$ satisfying

$$
\begin{gathered}
a\left(w_{h}, \psi\right)+b\left(\psi, p_{h}\right)=(f, \psi)_{D^{\prime}} \quad \forall \psi \in V_{h}, \\
b\left(w_{h}, v\right)=0, \quad \forall v \in W_{h} .
\end{gathered}
$$

As for the mixed finite element method for boundary value problems, Brezzi and Babuska, and others have established a systematic theory. Denote

$$
\begin{gathered}
V_{0}=\{u \in V: b(u, v)=0, \forall v \in W\}, \\
V_{h 0}=\left\{u \in V_{h}: b(u, v)=0, \forall v \in W_{h}\right\} .
\end{gathered}
$$

Theorem 2.1 (Brezzi-Babuska Theorem). Suppose that

(1) $a(\cdot, \cdot)$ and $b(\cdot, \cdot)$ are continuous bilinear forms, that is,

$$
\begin{gathered}
|a(q, \psi)| \leq M_{1}\|q\|_{V}\|\psi\|_{V^{\prime}} \quad \forall q, \psi \in V, \\
|b(\psi, v)| \leq M_{2}\|\psi\|_{V}\|v\|_{W}, \quad \forall \psi \in V, v \in W
\end{gathered}
$$


(2) there exists $v_{1}>0$, such that

$$
a(q, q) \geq v_{1}\|q\|_{V}^{2}, \quad \forall 0 \neq q \in V_{0}
$$

(3) inf-sup condition: there exists $v_{2}>0$, such that

$$
\sup _{\psi \in V, \psi \neq 0} \frac{b(\psi, v)}{\|\psi\|_{V}} \geq v_{2}\|v\|_{W}, \quad \forall v \in W
$$

then there exists a unique solution to the problem (2.7)-(2.8), and

$$
\|w\|_{V}+\|p\|_{W} \leq C\|f\|_{D^{\prime}}
$$

where constant $C$ just depends on $v_{1}, v_{2}$ and $M_{1}, M_{2}$. Furthermore, suppose that

(4) there exists a constant $\tilde{\mu}_{1}>0$, such that

$$
a(q, q) \geq \tilde{\mu}_{1}\|q\|_{V^{\prime}}^{2}, \quad \forall 0 \neq q \in V_{h 0}
$$

(5) discrete inf-sup condition: there exists a constant $\tilde{\mu}_{2}>0$, such that

$$
\sup _{\psi \in V_{h}, \psi \neq 0} \frac{b(\psi, v)}{\|\psi\|_{V}} \geq \tilde{\mu}_{2}\|v\|_{W}, \quad \forall v \in W_{h} .
$$

Then there exists a unique solution $\left(w_{h}, p_{h}\right)$ to the problem (2.9)-(2.10); moreover, the following error estimate is valid:

$$
\left\|w-w_{h}\right\|_{V}+\left\|p-p_{h}\right\|_{W} \leq C\left\{\inf _{q \in V_{h}}\|w-q\|_{V}+\inf _{v \in W_{h}}\|p-v\|_{W}\right\}
$$

where $C$ just depends on $\tilde{\mu}_{1}, \tilde{\mu}_{2}$ and $M_{1}, M_{2}$.

Since $a(\cdot, \cdot)$ is a symmetric, continuous, and $V$-elliptic bilinear form on $V \times V, b(\cdot, \cdot)$ is a continuous bilinear form, then conditions (1), (2), and (4) of Brezzi-Babuska Theorem hold. Suppose inf-sup condition and discrete inf-sup condition hold. Then by Brezzi-Babuska Theorem, we know that (2.7)-(2.8) and (2.9)-(2.10) are uniquely solvable for each $f \in D$. Thus we can define the corresponding linear bounded operators:

$T: D \rightarrow V, S: D \rightarrow W:$ for all $f \in D$

$$
\begin{gathered}
a(T f, \psi)+b(\psi, S f)=(f, \psi)_{D^{\prime}} \quad \forall \psi \in V, \\
b(T f, v)=0, \quad \forall v \in W,
\end{gathered}
$$


$T_{h}: D \rightarrow V_{h} \subset V, S_{h}: D \rightarrow W_{h} \subset W:$ for all $f \in D$,

$$
\begin{gathered}
a\left(T_{h} f, \psi\right)+b\left(\psi, S_{h} f\right)=(f, \psi)_{D^{\prime}}, \quad \forall \psi \in V_{h}, \\
b\left(T_{h} f, v\right)=0, \quad \forall v \in W_{h} .
\end{gathered}
$$

It is obvious that (2.3)-(2.4) have an equivalent operator form:

$$
\begin{gathered}
\lambda T u=u, \\
\sigma=S(\lambda u) .
\end{gathered}
$$

Equations (2.5)-(2.6) have an equivalent operator form:

$$
\begin{gathered}
\lambda_{h} T_{h} u_{h}=u_{h}, \\
\sigma_{h}=S_{h}\left(\lambda_{h} u_{h}\right) .
\end{gathered}
$$

It is easy to prove that $T: D \rightarrow D, T_{h}: D \rightarrow D$ are self-adjoint operators. In fact, for all $f, g \in D$, taking $\psi=T g, v=S g$ in (2.7)-(2.8) we obtain

$$
\begin{gathered}
a(T f, T g)+b(T g, S f)=(f, T g)_{D^{\prime}} \\
b(T f, S g)=0 .
\end{gathered}
$$

Exchanging $f$ and $g$, we get

$$
\begin{gathered}
a(T g, T f)+b(T f, S g)=(g, T f)_{D^{\prime}} \\
b(T g, S f)=0,
\end{gathered}
$$

then

$$
\begin{aligned}
(f, T g)_{D} & =a(T f, T g)+b(T g, S f)+b(T f, S g) \\
& =a(T g, T f)+b(T f, S g)+b(T g, S f)=(g, T f)_{D}+0=(T f, g)_{D} .
\end{aligned}
$$

It shows that $T: D \rightarrow D$ is self-adjoint in the sense of inner product $(\cdot, \cdot)_{D}$. Analogously, it can be proved that $T_{h}: D \rightarrow D$ is self-adjoint in the sense of inner product $(\cdot, \cdot)_{D}$.

Assume that $V \hookrightarrow D$ (compact imbedded), then it is easy to prove that the operator $T: D \rightarrow D$ is completely continuous, $T: V \rightarrow V$ is completely continuous, and $T_{h}$ is a finite rank operator. Combining (2.3)-(2.4), (2.5)-(2.6), and the $V$-ellipticity of $a(\cdot, \cdot)$, we deduce

$$
\lambda=\frac{a(u, u)}{(u, u)_{D}}>0, \quad \lambda_{h}=\frac{a\left(u_{h}, u_{h}\right)}{\left(u_{h}, u_{h}\right)_{D}}>0
$$


Then, from the spectral theory of self-adjoint and completely continuous operator we know that the eigenvalues of (2.3)-(2.4) can be sorted as

$$
0<\lambda_{1} \leq \lambda_{2} \leq \cdots \leq \lambda_{k} \leq \cdots \nearrow+\infty
$$

and the corresponding eigenfunctions are

$$
u_{1}, \sigma_{1}, u_{2}, \sigma_{2}, \ldots, u_{k}, \sigma_{k}, \ldots
$$

where $\left(u_{i}, u_{j}\right)_{D}=\delta_{i j}$.

The eigenvalues of (2.5)-(2.6) can be sorted as

$$
0<\lambda_{1, h} \leq \lambda_{2, h} \leq \cdots \leq \lambda_{k, h} \leq \cdots \leq \lambda_{N_{h}, h}
$$

and the corresponding eigenfunctions are

$$
u_{1, h}, \sigma_{1, h}, u_{2, h}, \sigma_{2, h}, \ldots, u_{k, h}, \sigma_{k, h}, \ldots, u_{N_{h}, h}, \sigma_{N_{h}, h}
$$

where $\left(u_{i, h}, u_{j, h}\right)_{D}=\delta_{i j}$.

It is obvious that $a(\cdot, \cdot)$ is an inner product on $V,\|\cdot\|_{a}=\sqrt{a(\cdot, \cdot)}$ and $\|\cdot\|_{V}$ are equivalent norms. Let $u_{h}=u_{i, h}$ in (2.5); then

$$
a\left(u_{i, h}, u_{j, h}\right)+b\left(u_{j, h}, \sigma_{h}\right)=\lambda_{i, h}\left(u_{i, h}, u_{j, h}\right)_{D}
$$

From (2.6), we get $b\left(u_{j, h}, \sigma_{h}\right)=0$. Then

$$
a\left(u_{i, h}, u_{j, h}\right)=\lambda_{i, h}\left(u_{i, h}, u_{j, h}\right)_{D}=\lambda_{i, h} \delta_{i j}
$$

therefore, $\left\{u_{i, h} /\left\|u_{i, h}\right\|_{a}\right\}$ is a completely normal eigenvector system on $V_{h}$ in the sense of inner product $a(\cdot, \cdot)$.

Denote $\lambda_{k}=1 / \mu_{k}, \lambda_{k, h}=1 / \mu_{k, h}$. In this paper, $\mu_{k}$ and $\mu_{k, h}, \lambda_{k}$, and $\lambda_{k, h}$ are all called eigenvalues. Let $\mu$ be the $k$ th eigenvalue with algebraic multiplicity $q, \mu=\mu_{k}=\mu_{k+1}=$ $\cdots=\mu_{k+q-1} \cdot M(\mu)$ is the space spanned by all eigenfunctions $\left\{u_{j}\right\}_{k}^{k+q-1}$ corresponding to $\mu$ of $T . M_{h}(\mu)$ is the space spanned by all eigenfunctions $\left\{u_{j, h}\right\}_{k}^{k+q-1}$ corresponding to all eigenvalues of $T_{h}$ that converge to $\mu$. Let $\widehat{M}(\mu)=\left\{v: v \in M(\mu),\|v\|_{a}=1\right\}, \widehat{M}_{h}(\mu)=\{v$ : $\left.v \in M_{h}(\mu),\|v\|_{a}=1\right\}$. We call $\lambda=1 / \mu$ the $k$ th eigenvalue, too. Denote $M(\lambda)=M(\mu)$, $M_{h}(\lambda)=M_{h}(\mu)$, and $\widehat{M}(\lambda)=\widehat{M}(\mu)$. Define

$$
\begin{aligned}
& \left\|\left.\left(T-T_{h}\right)\right|_{M(\lambda)}\right\|_{D}=\max _{u \in M(\lambda), u \neq 0} \frac{\left\|\left(T-T_{h}\right) u\right\|_{D}}{\|u\|_{D}}, \\
& \left\|\left.\left(T-T_{h}\right)\right|_{M(\lambda)}\right\|_{a}=\max _{u \in M(\lambda), u \neq 0} \frac{\left\|\left(T-T_{h}\right) u\right\|_{a}}{\|u\|_{a}} .
\end{aligned}
$$


The convergence and error estimate about mixed element method of eigenvalue problem have been studied by [23-25]. From these literatures we easily know that the following results are valid.

Lemma 2.2. Suppose that the conditions of Brezzi-Babuska Theorem hold, and $\left\|T-T_{h}\right\|_{D} \rightarrow 0(h \rightarrow$ $0)$. Let $\left(\lambda_{h}, u_{h}, \sigma_{h}\right)$ be the $k$ th eigenpair of (2.5)-(2.6), $\left\|u_{h}\right\|_{a}=1$, and $\lambda$ the $k$ th eigenvalue of (2.3)(2.4). Then $\lambda_{h} \rightarrow \lambda(h \rightarrow 0)$, and there exists an eigenfunction $(u, \sigma)$ corresponding to $\lambda$ such that

$$
\begin{gathered}
\left|\lambda_{h}-\lambda\right|+\left\|u_{h}-u\right\|_{D} \leq C\left\|\left.\left(T-T_{h}\right)\right|_{M(\lambda)}\right\|_{D^{\prime}} \\
\left\|\sigma-\sigma_{h}\right\|_{W} \leq\left\|S_{h}(\lambda u)-S(\lambda u)\right\|_{W}+C\left\|\left.\left(T-T_{h}\right)\right|_{M(\lambda)}\right\|_{D^{\prime}} \\
\left\|u-u_{h}\right\|_{a} \leq C\left\|\left.\left(T_{h}-T\right)\right|_{M(\lambda)}\right\|_{a}
\end{gathered}
$$

Let $u \in \widehat{M}(\lambda)$; then there exists $u_{h} \in M_{h}(\lambda)$ such that

$$
\left\|u-u_{h}\right\|_{a} \leq C\left\|\left.\left(T_{h}-T\right)\right|_{M(\lambda)}\right\|_{a}
$$

Proof. From the spectral approximation theory (see [23]) we have (2.39).

Let $u$ satisfy (2.39), and $\sigma=S(\lambda u)$. Next we will prove that this eigenpair satisfies (2.40)-(2.41). From Brezzi-Babuska Theorem and (2.39), we get

$$
\begin{gathered}
\left\|S\left(\lambda u-\lambda_{h} u_{h}\right)\right\|_{W} \leq C\left\|\lambda u-\lambda_{h} u_{h}\right\|_{D} \leq C\left\|\left.\left(T-T_{h}\right)\right|_{M(\lambda)}\right\|_{D^{\prime}} \\
\left\|T\left(\lambda u-\lambda_{h} u_{h}\right)\right\|_{V} \leq C\left\|\lambda u-\lambda_{h} u_{h}\right\|_{D} \leq C\left\|\left.\left(T-T_{h}\right)\right|_{M(\lambda)}\right\|_{D} .
\end{gathered}
$$

Using the triangle inequality and (2.43), we deduce

$$
\begin{aligned}
\left\|\sigma-\sigma_{h}\right\|_{W}-\left\|S_{h}(\lambda u)-S(\lambda u)\right\|_{W} \\
\quad=\left|\left\|S(\lambda u)-S_{h}\left(\lambda_{h} u_{h}\right)\right\|_{W}-\left\|S_{h}(\lambda u)-S(\lambda u)\right\|_{W}\right| \\
\leq\left\|S_{h}\left(\lambda u-\lambda_{h} u_{h}\right)\right\|_{W} \leq C\left\|\left.\left(T-T_{h}\right)\right|_{M(\lambda)}\right\|_{D^{\prime}}
\end{aligned}
$$

that is, $(2.40)$ is valid.

Using the triangle inequality and (2.44), we get

$$
\begin{aligned}
\left\|u-u_{h}\right\|_{a}-\left\|T_{h}(\lambda u)-T(\lambda u)\right\|_{a} \\
\quad=\left|\left\|T(\lambda u)-T_{h}\left(\lambda_{h} u_{h}\right)\right\|_{a}-\left\|T_{h}(\lambda u)-T(\lambda u)\right\|_{a}\right| \\
\leq\left\|T_{h}\left(\lambda u-\lambda_{h} u_{h}\right)\right\|_{a} \\
\quad \leq C\left\|\left.\left(T-T_{h}\right)\right|_{M(\lambda)}\right\|_{D} \leq C\left\|\left.\left(T-T_{h}\right)\right|_{M(\lambda)}\right\|_{a^{\prime}}
\end{aligned}
$$

which together with $\left\|T_{h}(\lambda u)-T(\lambda u)\right\|_{a} \leq C\left\|\left.\left(T-T_{h}\right)\right|_{M(\lambda)}\right\|_{a}$ yields (2.41). 
Let the eigenfunctions $\left\{u_{j, h}\right\}$ be an orthonormal system of $M_{h}(\lambda)$ in the sense of inner product $a(\cdot, \cdot)$. Then, from (2.41) and Lemma 3.1 we know that there exists a basis $\left\{u_{j}^{0}\right\}$ of $M(\lambda)$ satisfying $\left\|u_{j}^{0}\right\|_{a}=1$ and the following result is valid:

$$
\left\|u_{j}^{0}-u_{j, h}\right\|_{a} \leq C\left\|\left.\left(T_{h}-T\right)\right|_{M(\lambda)}\right\|_{a} .
$$

For any $u \in \widehat{M}(\lambda)$, we write $u=\sum_{j=k}^{k+q-1} \alpha_{j} u_{j}^{0}$. By calculation, we get

$$
1=\|u\|_{a}^{2}=\sum_{j=k}^{k+q-1} \alpha_{j}^{2}+\sum_{i \neq j, i, j=k}^{k+q-1} \alpha_{i} \alpha_{j} a\left(u_{i}^{0}, u_{j}^{0}\right) .
$$

From (2.47), when $i \neq j$ we have $a\left(u_{i}^{0}, u_{j}^{0}\right)=a\left(u_{i}^{0}, u_{j}^{0}\right)-a\left(u_{i, h}, u_{j, h}\right) \rightarrow 0(h \rightarrow 0)$, and thus we get $\sum_{j=k}^{k+q-1} \alpha_{j}^{2} \rightarrow 1(h \rightarrow 0)$.

Denote $u_{h}=\sum_{j=k}^{k+q-1} \alpha_{j} u_{j, h}$, then $u_{h} \in M_{h}(\lambda)$. From (2.47), we deduce

$$
\left\|u-u_{h}\right\|_{a} \leq C \sum_{j=k}^{k+q-1}\left\|u_{j, h}-u_{j}^{0}\right\|_{a} \leq C\left\|\left.\left(T_{h}-T\right)\right|_{M(\curlywedge)}\right\|_{a^{\prime}}
$$

that is, (2.42) is valid. The proof is completed.

For $\left(u^{*}, \sigma^{*}\right) \in V \times W, u^{*} \neq 0$, define the Rayleigh quotient

$$
\lambda^{r}=\frac{a\left(u^{*}, u^{*}\right)+2 b\left(u^{*}, \sigma^{*}\right)}{\left(u^{*}, u^{*}\right)_{D}} .
$$

The following lemma is an extension of [23, Lemma 9.1].

Lemma 2.3. Let $(\lambda, u, \sigma)$ be an eigenpair of (2.3)-(2.4); then for all $\left(u^{*}, \sigma^{*}\right) \in V \times W,\left(u^{*}, \sigma^{*}\right) \neq(0,0)$ with its Rayleigh quotient satisfying

$$
\lambda^{r}-\lambda=\frac{a\left(u^{*}-u, u^{*}-u\right)+2 b\left(u^{*}-u, \sigma^{*}-\sigma\right)}{\left(u^{*}, u^{*}\right)_{D}}-\lambda \frac{\left(u^{*}-u, u^{*}-u\right)_{D}}{\left(u^{*}, u^{*}\right)_{D}} .
$$


Proof. From (2.3)-(2.4), we deduce

$$
\begin{aligned}
a\left(u^{*}-\right. & \left.u, u^{*}-u\right)+2 b\left(u^{*}-u, \sigma^{*}-\sigma\right)-\lambda\left(u^{*}-u, u^{*}-u\right)_{D} \\
= & a\left(u^{*}, u^{*}\right)-2 a\left(u^{*}, u\right)+a(u, u)+2 b\left(u^{*}, \sigma^{*}\right)-2 b\left(u^{*}, \sigma\right)-2 b\left(u, \sigma^{*}\right) \\
& +2 b(u, \sigma)-\lambda\left(u^{*}, u^{*}\right)_{D}+2 \lambda\left(u^{*}, u\right)_{D}-\lambda(u, u)_{D} . \\
= & a\left(u^{*}, u^{*}\right)+2 b\left(u^{*}, \sigma^{*}\right)-\lambda\left(u^{*}, u^{*}\right)_{D}-2\left(a\left(u^{*}, u\right)+b\left(u^{*}, \sigma\right)-\lambda\left(u^{*}, u\right)_{D}\right) \\
& -2 b\left(u, \sigma^{*}\right)+\left(a(u, u)+b(u, \sigma)-\lambda(u, u)_{D}\right)+b(u, \sigma) . \\
= & a\left(u^{*}, u^{*}\right)+2 b\left(u^{*}, \sigma^{*}\right)-\lambda\left(u^{*}, u^{*}\right)_{D}-0-0+0+0 .
\end{aligned}
$$

By dividing by $\left(u^{*}, u^{*}\right)_{D}$ on both sides of the above identity, we obtain (2.51).

Taking $\left(u^{*}, \sigma^{*}\right)=\left(u_{h}, \sigma_{h}\right)$ in (2.51) and using (2.4) and (2.6), we derive

Lemma 2.4. Let $(\lambda, u, \sigma)$ and $\left(\lambda_{h}, u_{h}, \sigma_{h}\right)$ be the kth eigenpair of (2.3)-(2.4) and (2.5)-(2.6), respectively; then

$$
\begin{gathered}
\lambda_{h}-\lambda=\frac{a\left(u_{h}-u, u_{h}-u\right)+2 b\left(u_{h}-u, v-\sigma\right)}{\left(u_{h}, u_{h}\right)_{D}}-\lambda \frac{\left(u_{h}-u, u_{h}-u\right)_{D}}{\left(u_{h}, u_{h}\right)_{D}}, \quad \forall v \in W_{h}, \\
\left|\lambda_{h}-\lambda\right| \leq C\left(\left\|u_{h}-u\right\|_{a}^{2}+\left\|u_{h}-u\right\|_{a}\|\sigma-v\|_{W}\right), \quad \forall v \in W_{h} .
\end{gathered}
$$

\section{The Two-Scale Discretization Scheme for Mixed Variational Formulation of Eigenvalue Problems}

This paper establishes the following finite element two-scale discretization scheme based on the shifted-inverse power method.

Scheme 1. One has the following.

Step 1. Solve the eigenvalue problem (2.3)-(2.4) on a coarse grid $K^{H}$ : find $\left(\lambda_{H}, u_{H}, \sigma_{H}\right) \in \mathbf{R} \times V_{H} \times W_{H},\left\|u_{H}\right\|_{a}=1$ such that

$$
\begin{gathered}
a\left(u_{H}, \psi\right)+b\left(\psi, \sigma_{H}\right)=\lambda_{H}\left(u_{H}, \psi\right)_{D^{\prime}} \quad \forall \psi \in V_{H}, \\
b\left(u_{H}, v\right)=0, \quad \forall v \in W_{H} .
\end{gathered}
$$

Step 2. Solve a equation on a fine grid $K^{h}$ : find $\left(u^{\prime}, \sigma^{\prime}\right) \in V_{h} \times W_{h}$ such that

$$
\begin{gathered}
a\left(u^{\prime}, \psi\right)+b\left(\psi, \sigma^{\prime}\right)-\lambda_{H}\left(u^{\prime}, \psi\right)_{D}=\left(u_{H}, \psi\right)_{D^{\prime}} \quad \forall \psi \in V_{h}, \\
b\left(u^{\prime}, v\right)=0, \quad \forall v \in W_{h} .
\end{gathered}
$$

Set $u^{h}=u^{\prime} /\left\|u^{\prime}\right\|_{a}, \sigma^{h}=\sigma^{\prime} /\left\|u^{\prime}\right\|_{a}$. 
Step 3. Compute the Rayleigh quotient

$$
\lambda^{h}=\frac{a\left(u^{h}, u^{h}\right)}{\left(u^{h}, u^{h}\right)_{D}} .
$$

Next we will discuss the validity of Scheme 1.

Lemma 3.1. For any nonzero elements $u, v \in V$,

$$
\left\|\frac{u}{\|u\|_{a}}-\frac{v}{\|v\|_{a}}\right\|_{a} \leq 2 \frac{\|u-v\|_{a}}{\|u\|_{a}}, \quad\left\|\frac{u}{\|u\|_{a}}-\frac{v}{\|v\|_{a}}\right\|_{a} \leq 2 \frac{\|u-v\|_{a}}{\|v\|_{a}} .
$$

Proof. See [21].

Denote $\operatorname{dist}(u, V)=\inf _{v \in V}\|u-v\|_{a}$.

Consider the eigenvalue problem (2.25) on the space $V_{h}$.

Lemma 3.2. Suppose that $\mu$ and $\mu_{h}$ are the $k$ th eigenvalue of $T$ and $T_{h}$, respectively, and $\left(\mu_{0}, u_{0}\right)$ is an approximate eigenpair where $\mu_{0}$ is not an eigenvalue of $T_{h}, u_{0} \in V_{h},\left\|u_{0}\right\|_{a}=1, \operatorname{dist}\left(u_{0}, M_{h}(\mu)\right) \leq$ $1 / 2, \max _{k \leq j \leq k+q-1}\left|\left(\mu_{j, h}-\mu_{h}\right) /\left(\mu_{0}-\mu_{j, h}\right)\right| \leq 1 / 2,\left|\mu_{0}-\mu_{j, h}\right| \geq(\rho / 2)(j \neq k, k+1, \ldots, k+q-1)$, and $u^{s} \in V_{h}, u^{h} \in V_{h}$ satisfy

$$
\left(\mu_{0}-T_{h}\right) u^{s}=u_{0}, \quad u^{h}=\frac{u^{s}}{\left\|u^{s}\right\|_{a}} .
$$

Then

$$
\operatorname{dist}\left(u^{h}, \widehat{M}_{h}(\mu)\right) \leq \frac{16}{\rho}\left|\mu_{0}-\mu_{h}\right| \operatorname{dist}\left(u_{0}, M_{h}(\mu)\right),
$$

where $\rho=\min _{\mu_{j} \neq \mu}\left|\mu_{j}-\mu\right|$ is the separation constant of the eigenvalue $\mu$.

Proof. See [21].

Theorem 3.3. Suppose that the conditions of Brezzi-Babuska Theorem hold and $\left\|T_{h}-T\right\|_{D} \rightarrow 0(h \rightarrow$ $0)$. Let $\left(\lambda^{h}, u^{h}, \sigma^{h}\right)$ be the approximate eigenpair obtained by the two-scale discretization scheme and $H$ small properly. Then there exists $u \in M(\lambda)$ such that

$$
\begin{gathered}
\left\|u^{h}-u\right\|_{a} \leq C\left(\left|\lambda_{H}-\lambda\right|^{2}+\left|\lambda_{H}-\lambda\right|\left\|\left.\left(T-T_{H}\right)\right|_{M(\lambda)}\right\|_{D}+\left\|\left.\left(T-T_{h}\right)\right|_{M(\lambda)}\right\|_{a}\right), \\
\left|\lambda^{h}-\lambda\right| \leq C\left(\left\|u^{h}-u\right\|_{a}^{2}+\left\|u^{h}-u\right\|_{a} \inf _{v \in W_{h}}\|\sigma-v\|_{W}\right) .
\end{gathered}
$$

Proof. We use Lemma 3.2 in the proof. 
Select $\mu_{0}=1 / \lambda_{H}$ and $u_{0}=\lambda_{H} T_{h} u_{H} /\left\|\lambda_{H} T_{h} u_{H}\right\|_{a}$. Let $u^{0} \in M(\lambda)$ such that $u_{H}-u^{0}$ satisfies (2.39) and (2.41). By calculation we deduce

$$
\begin{aligned}
\left\|\lambda_{H} T_{h} u_{H}-u^{0}\right\|_{a} & =\left\|\lambda_{H} T_{h} u_{H}-\lambda T u^{0}\right\|_{a} \\
& \leq C\left(\left|\lambda_{H}-\lambda\right|+\left\|u_{H}-u^{0}\right\|_{D}+\left\|\left(T-T_{h}\right) u^{0}\right\|_{a}\right) \\
& \leq C\left(\left|\lambda_{H}-\lambda\right|+\left\|\left.\left(T-T_{H}\right)\right|_{M(\lambda)}\right\|_{D}+\left\|\left.\left(T-T_{h}\right)\right|_{M(\lambda)}\right\|_{a}\right) ;
\end{aligned}
$$

thus, using Lemma 3.1, we get

$$
\begin{aligned}
\operatorname{dist}\left(u_{0}, \widehat{M}(\lambda)\right) & \leq\left\|u_{0}-\frac{u^{0}}{\left\|u^{0}\right\|_{a}}\right\|_{a} \leq C\left\|\lambda_{H} T_{h} u_{H}-u^{0}\right\|_{a} \\
& \leq C\left(\left|\lambda_{H}-\lambda\right|+\left\|\left.\left(T-T_{H}\right)\right|_{M(\lambda)}\right\|_{D}+\left\|\left.\left(T-T_{h}\right)\right|_{M(\lambda)}\right\|_{a}\right) .
\end{aligned}
$$

Using the triangle inequality and (2.42), we derive

$$
\operatorname{dist}\left(u_{0}, M_{h}(\lambda)\right) \leq \operatorname{dist}\left(u_{0}, \widehat{M}(\lambda)\right)+C\left\|\left.\left(T-T_{h}\right)\right|_{M(\lambda)}\right\|_{a} .
$$

From Lemma 2.2 we know $\lambda_{H} \rightarrow \lambda, \lambda_{j, h} \rightarrow \lambda$; then

$$
\left|\mu_{0}-\mu_{j, h}\right|=\left|\frac{\lambda_{H}-\lambda+\lambda-\lambda_{j, h}}{\lambda_{j, h} \lambda_{H}}\right| .
$$

When $H$ is small enough, noting that $h \ll H$, from (3.11) and (3.10) we get

$$
\operatorname{dist}\left(u_{0}, M_{h}(\lambda)\right) \leq \frac{1}{2}
$$

Having in mind that $\lambda=\lambda_{k+1}=\cdots=\lambda_{k+q-1}$ we have

$$
\left|\mu_{j, h}-\mu_{h}\right|=\left|\frac{\lambda_{h}-\lambda_{j, h}}{\lambda_{h} \lambda_{j, h}}\right|=\left|\frac{\lambda_{h}-\lambda+\lambda_{j}-\lambda_{j, h}}{\lambda_{h} \lambda_{j, h}}\right|,
$$

which together with (3.12), noting that $\lambda_{j, h}-\lambda$ is an infinitesimal of higher order comparing with $\lambda_{H}-\lambda$, yields

$$
\max _{k \leq j \leq k+q-1}\left|\frac{\mu_{j, h}-\mu_{h}}{\mu_{0}-\mu_{j, h}}\right| \leq \frac{1}{2} .
$$

Since $\rho$ is the separation constant, $H$ is small enough, and $h \ll H$, there holds

$$
\left|\mu_{0}-\mu_{j, h}\right| \geq \frac{\rho}{2}, \quad j \neq k, k+1, \ldots, k+q-1 .
$$


For $u^{\prime}$ in Step 2 of Scheme 1, from the definition of $T_{h}$ and $S_{h}$ we have

$$
\begin{gathered}
a\left(T_{h} \lambda_{H} u^{\prime}, \psi\right)+b\left(\psi, S_{h} \lambda_{H} u^{\prime}\right)=\lambda_{H}\left(u^{\prime}, \psi\right)_{D^{\prime}} \quad \forall \psi \in V_{h}, \\
b\left(T_{h} u^{\prime}, v\right)=0, \quad \forall v \in W_{h} . \\
a\left(T_{h} u_{H}, \psi\right)+b\left(\psi, S_{h} u_{H}\right)=\left(u_{H}, \psi\right)_{D^{\prime}} \quad \forall \psi \in V_{h}, \\
b\left(T_{h} u_{H}, v\right)=0, \quad \forall v \in W_{h} .
\end{gathered}
$$

Hence, Step 2 of Scheme 1 is equivalent to $\left(u^{\prime}, \sigma^{\prime}\right) \in V_{h} \times W_{h}$,

$$
\begin{gathered}
a\left(u^{\prime}, \psi\right)+b\left(\psi, \sigma^{\prime}\right)-\lambda_{H} a\left(T_{h} u^{\prime}, \psi\right)-\lambda_{H} b\left(\psi, S_{h} u^{\prime}\right)=a\left(T_{h} u_{H}, \psi\right)+b\left(\psi, S_{h} u_{H}\right), \quad \forall \psi \in V_{h}, \\
b\left(u^{\prime}, v\right)=0, \quad \forall v \in W_{h}
\end{gathered}
$$

$u^{h}=u^{\prime} /\left\|u^{\prime}\right\|_{a}, \sigma^{h}=\sigma^{\prime} /\left\|u^{\prime}\right\|_{a}$.

From (3.21) we obtain

$$
a\left(u^{\prime}-\lambda_{H} T_{h} u^{\prime}-T_{h} u_{H}, \psi\right)+b\left(\psi, \sigma^{\prime}-\lambda_{H} S_{h} u^{\prime}-S_{h} u_{H}\right)=0, \quad \forall \psi \in V_{h}
$$

Combining (3.22), (3.18), and (3.20), we get

$$
b\left(u^{\prime}-\lambda_{H} T_{h} u^{\prime}-T_{h} u_{H}, v\right)=0, \quad \forall v \in W_{h} .
$$

By (3.24), taking $\psi=u^{\prime}-\lambda_{H} T_{h} u^{\prime}-T_{h} u_{H}$ in (3.23), we obtain

$$
a\left(u^{\prime}-\lambda_{H} T_{h} u^{\prime}-T_{h} u_{H}, u^{\prime}-\lambda_{H} T_{h} u^{\prime}-T_{h} u_{H}\right)=0 .
$$

Thus

$$
\left(\frac{1}{\lambda_{H}}-T_{h}\right) u^{\prime}=\frac{1}{\lambda_{H}} T_{h} u_{H}, \quad u^{h}=\frac{u^{\prime}}{\left\|u^{\prime}\right\|_{a}}
$$

From (3.26) we know that the first term on the left-hand side of (3.23) is equal to 0; thus

$$
b\left(\psi, \sigma^{\prime}-\lambda_{H} S_{h} u^{\prime}-S_{h} u_{H}\right)=0, \quad \forall \psi \in V_{h} ;
$$

then, using discrete inf-sup condition, we obtain

$$
\sigma^{\prime}=\lambda_{H} S_{h} u^{\prime}+S_{h} u_{H}
$$


Thus Step 2 of Scheme 1 is equivalent to (3.26), (3.28), and $u^{h}=u^{\prime} /\left\|u^{\prime}\right\|_{a}, \sigma^{h}=\sigma^{\prime} /\left\|u^{\prime}\right\|_{a}$. Noting that $\lambda_{H}^{-1} T_{h} u_{H}=\left\|\lambda_{H}^{-1} T_{h} u_{H}\right\|_{a} u_{0}$ differs from $u_{0}$ by only a constant and denoting $u^{s}=$ $u^{\prime} /\left\|\lambda_{H}^{-1} T_{h} u_{H}\right\|_{a}$, we have

$$
\left(\frac{1}{\lambda_{H}}-T_{h}\right) u^{s}=u_{0}, \quad u^{h}=\frac{u^{s}}{\left\|u^{s}\right\|_{a}} .
$$

By (3.13), (3.15), (3.16), and (3.29), we see that the conditions of Lemma 3.2 hold. Thus, substituting (3.11) and (3.12) into (3.6), we obtain

$$
\operatorname{dist}\left(u^{h}, \widehat{M}_{h}(\lambda)\right) \leq C\left|\lambda_{H}-\lambda\right|\left(\operatorname{dist}\left(u_{0}, \widehat{M}(\lambda)\right)+\left\|T u-T_{h} u\right\|_{a}\right)
$$

Let the eigenfunctions $\left\{u_{j, h}\right\}_{k}^{k+q-1}$ be an orthonormal system of $M_{h}(\lambda)$ (in the sense of $a(\cdot, \cdot)$ ). Then

$$
\operatorname{dist}\left(u^{h}, M_{h}(\lambda)\right)=\left\|u^{h}-\sum_{j=k}^{k+q-1} a\left(u^{h}, u_{j, h}\right) u_{j, h}\right\|_{a} .
$$

Let

$$
u^{*}=\sum_{j=k}^{k+q-1} a\left(u^{h}, u_{j, h}\right) u_{j, h}
$$

and noting that $\left\|u^{h}-u^{*}\right\|_{a} \leq \operatorname{dist}\left(u^{h}, \widehat{M}_{h}(\lambda)\right)$, from (3.30) we deduce

$$
\left\|u^{h}-u^{*}\right\|_{a} \leq C\left|\lambda_{H}-\lambda\right|\left(\operatorname{dist}\left(u_{0}, \widehat{M}(\lambda)\right)+\left\|T u-T_{h} u\right\|_{a}\right) .
$$

By Lemma 2.2, there exists $\left\{u_{j}^{0}\right\}_{k}^{k+q-1} \subset M(\lambda)$ such that $u_{j, h}-u_{j}^{0}$ satisfies (2.41). Let

$$
u=\sum_{j=k}^{k+q-1} a\left(u^{h}, u_{j, h}\right) u_{j}^{0}
$$

then $u \in M(\lambda)$. Using (2.41) we deduce

$$
\begin{aligned}
\left\|u^{*}-u\right\|_{a} & =\left\|\sum_{j=k}^{k+q-1} a\left(u^{h}, u_{j, h}\right)\left(u_{j, h}-u_{j}^{0}\right)\right\|_{a} \\
& \leq C\left(\sum_{j=k}^{k+q-1}\left\|u_{j, h}-u_{j}^{0}\right\|_{a}^{2}\right)^{1 / 2} \leq C\left\|\left.\left(T_{h}-T\right)\right|_{M(\lambda)}\right\|_{a} .
\end{aligned}
$$


Combining (3.33) with the previous inequality, we have

$$
\left\|u^{h}-u\right\|_{a} \leq C\left(\left|\lambda_{H}-\lambda\right| \operatorname{dist}\left(u_{0}, \widehat{M}(\lambda)\right)+\left\|\left.\left(T_{h}-T\right)\right|_{M(\lambda)}\right\|_{a}\right) .
$$

Substituting (3.10) into (3.36), we get (3.7).

We know that $b\left(u^{h}, \sigma^{h}\right)=0$ from Step 2 of two-scale scheme; then

$$
\lambda^{h}=\frac{a\left(u^{h}, u^{h}\right)}{\left(u^{h}, u^{h}\right)_{D}}=\frac{a\left(u^{h}, u^{h}\right)+2 b\left(u^{h}, \sigma^{h}\right)}{\left(u^{h}, u^{h}\right)_{D}} .
$$

Select $\lambda^{r}=\lambda^{h}, u^{*}=u^{h}, \sigma^{*}=\sigma^{h}$. From Lemma 2.3, we get

$$
\lambda^{h}-\lambda=\frac{a\left(u^{h}-u, u^{h}-u\right)+2 b\left(u^{h}-u, \sigma^{h}-\sigma\right)}{\left(u^{h}, u^{h}\right)_{D}}-\lambda \frac{\left(u^{h}-u, u^{h}-u\right)_{D}}{\left(u^{h}, u^{h}\right)_{D}} .
$$

Noting that, for all $v \in W_{h}, b\left(u^{h}-u, v\right)=0$, we have

$$
\lambda^{h}-\lambda=\frac{a\left(u^{h}-u, u^{h}-u\right)+2 b\left(u^{h}-u, v-\sigma\right)}{\left(u^{h}, u^{h}\right)_{D}}-\lambda \frac{\left(u^{h}-u, u^{h}-u\right)_{D}}{\left(u^{h}, u^{h}\right)_{D}}, \quad \forall v \in W_{h} .
$$

Since $V \hookrightarrow D$ (continuously imbedded), $\left\|u^{h}-u\right\|_{D} \leq C\left\|u^{h}-u\right\|_{a}$. Then from (3.39) we obtain (3.8).

\section{Two-Scale Discretization Scheme for Stokes Eigenvalue Problem}

Consider the Stokes eigenvalue problem:

$$
\begin{gathered}
-\Delta \vec{u}+\nabla \sigma=\lambda \vec{u}, \quad \text { in } \Omega, \\
\operatorname{div} \vec{u}=0, \quad \text { in } \Omega, \\
\vec{u}=0, \quad \text { on } \partial \Omega,
\end{gathered}
$$

where $\Omega$ is a polygonal domain in $\mathbf{R}^{2}, \vec{u}=\left(u_{1}, u_{2}\right)$ denotes the fluid velocity, and $\sigma$ denotes the pressure.

In this paper, we use the symbol $\rightarrow$ to stand for vector function. For the function $\sigma$ in $H^{m}(\Omega)$, let

$$
\|\sigma\|_{m}=\left(\sum_{|\alpha| \leq m} \int_{\Omega}\left|\partial^{\alpha} \sigma\right|^{2}\right)^{1 / 2}, \quad \alpha=\left\{\alpha_{1}, \alpha_{2}\right\},|\alpha|=\alpha_{1}+\alpha_{2} .
$$


For vector function $\vec{u}=\left(u_{1}, u_{2}\right)$, define

$$
\|\vec{u}\|_{m}=\left(\left\|u_{1}\right\|_{m}^{2}+\left\|u_{2}\right\|_{m}^{2}\right)^{1 / 2}, \quad|\vec{u}|_{m}=\left(\left|u_{1}\right|_{m}^{2}+\left|u_{2}\right|_{m}^{2}\right)^{1 / 2}
$$

Using Green's formula, we derive the mixed variational form associated with (4.1)(4.3).

Find $(\lambda, \vec{u}, \sigma) \in \mathbf{R} \times H_{0}^{1}(\Omega)^{2} \times L_{0}^{2}(\Omega)$ with $|\vec{u}|_{1}=1$ such that

$$
\begin{gathered}
\int_{\Omega} \sum_{i=1}^{2} \nabla u_{i} \cdot \nabla \psi_{i}-\int_{\Omega} \operatorname{div} \vec{\psi} \sigma=\lambda \int_{\Omega} \vec{u} \cdot \vec{\psi}, \quad \forall \vec{\psi} \in H_{0}^{1}(\Omega)^{2} \\
-\int_{\Omega} \operatorname{div} \vec{u} v=0, \quad \forall v \in L_{0}^{2}(\Omega) .
\end{gathered}
$$

Let $V_{h} \subset H_{0}^{1}(\Omega)^{2}, W_{h} \subset L_{0}^{2}(\Omega)$ be two mixed finite element spaces. The mixed finite element form is as follows.

Seek $\left(\lambda_{h}, \vec{u}_{h}, \sigma_{h}\right) \in \mathbf{R} \times V_{h} \times W_{h}$ with $\left|\vec{u}_{h}\right|_{1}=1$ such that

$$
\begin{gathered}
\int_{\Omega} \sum_{i=1}^{2} \nabla u_{h i} \cdot \nabla \psi_{i}-\int_{\Omega} \operatorname{div} \vec{\psi}_{h} \sigma_{h}=\lambda \int_{\Omega} \vec{u}_{h} \cdot \vec{\psi}, \quad \forall \vec{\psi} \in V_{h} \\
-\int_{\Omega} \operatorname{div} \vec{u}_{h} v=0, \quad \forall v \in W_{h} .
\end{gathered}
$$

Denote

$$
\begin{gathered}
V=H_{0}^{1}(\Omega) \times H_{0}^{1}(\Omega), \\
W=L_{0}^{2}(\Omega)=\left\{v \in L^{2}(\Omega): \int_{\Omega} v=0\right\}, \quad D=L^{2}(\Omega) \times L^{2}(\Omega), \\
a(\vec{u}, \vec{\psi})=\int_{\Omega} \sum_{i=1}^{2} \nabla u_{i} \cdot \nabla \psi_{i}, \\
b(\vec{u}, v)=-\int_{\Omega} \operatorname{div} \vec{u} v, \quad(\vec{u}, \vec{\psi})_{D}=\int_{\Omega} \vec{u} \cdot \vec{\psi} .
\end{gathered}
$$

Let $\|\vec{u}\|_{a}=\sqrt{a(\vec{u}, \vec{u})}$. It is clear that $\|\vec{u}\|_{a}=|\vec{u}|_{1}$ is a norm. Then (4.6)-(4.7) and (4.8)-(4.9) can be written in the forms of (2.3)-(2.4) and (2.5)-(2.6), respectively (we need to add $\rightarrow$ for the vector function, e.g., $u, \psi$ should be written in the forms of $\vec{u}, \vec{\psi})$.

We apply Scheme 1 to the Stokes eigenvalue problem (4.6)-(4.7). Adding the symbol $\rightarrow$ for the vector function we get two-scale discretization scheme of mixed finite element for solving the Stokes eigenvalue problem (4.6)-(4.7), which is still called Scheme 1.

Consider the associated source and approximate source problems. 
Find $(\vec{w}, p) \in V \times W$ such that

$$
\begin{gathered}
a(\vec{w}, \vec{\psi})+b(\vec{\psi}, p)=(\vec{f}, \vec{\psi})_{D^{\prime}} \quad \forall \vec{\psi} \in V, \\
b(\vec{w}, v)=0, \quad \forall v \in W .
\end{gathered}
$$

Seek $\left(\vec{w}_{h}, p_{h}\right) \in V_{h} \times W_{h}$ such that

$$
\begin{gathered}
a\left(\vec{w}_{h}, \vec{\psi}\right)+b\left(\vec{\psi}, p_{h}\right)=(\vec{f}, \vec{\psi})_{D^{\prime}} \quad \forall \vec{\psi} \in V_{h \prime} \\
b\left(\vec{w}_{h}, v\right)=0, \quad \forall v \in W_{h} .
\end{gathered}
$$

From [26] we know that (4.11)-(4.12) satisfy conditions (1)-(3) in Brezzi-Babuska Theorem; therefore, there exists a unique solution $(\vec{w}, p) \in V \times W$ to the problem (4.11)-(4.12) and the following estimate is valid:

$$
\|\vec{w}\|_{1}+\|p\|_{0} \leq C_{p}\|\vec{f}\|_{0}
$$

Condition (4) in Brezzi-Babuska Theorem holds since $V_{h} \subset V$ and $W_{h} \subset W$. Suppose that condition (5) in Brezzi-Babuska Theorem (discrete inf-sup condition) is valid, then there exists a unique solution $\left(\vec{w}_{h}, p_{h}\right) \in V_{h} \times W_{h}$ to the problem (4.13)-(4.14), and the following error estimate is valid (see [27-29]):

$$
\left\|\vec{w}-\vec{w}_{h}\right\|_{1}+\left\|p-p_{h}\right\|_{0} \leq C\left(\inf _{\vec{\psi} \in V_{h}}\|\vec{w}-\vec{\psi}\|_{1}+\inf _{v \in W_{h}}\|p-v\|_{0}\right) .
$$

We assume that the following a prior estimate holds: for any $\vec{f} \in D, \vec{w} \in H^{1+r}(\Omega) \times$ $H^{1+r}(\Omega), p \in H^{r}(\Omega)$, and

$$
\|\vec{w}\|_{1+r}+\|p\|_{r} \leq C\|\vec{f}\|_{0^{\prime}}
$$

where $0<r \leq 1$ is a number determined by the maximal inner angle $\omega$ of $\Omega$. When $\omega<\pi$, $r=1$ (see [30]).

Suppose that the following estimate holds: for any $\vec{w} \in H^{1+r}(\Omega) \times H^{1+r}(\Omega)$, and for any $p \in H^{r}(\Omega)$,

$$
\inf _{\vec{\psi} \in V_{h}}\|\vec{w}-\vec{\psi}\|_{V}+\inf _{v \in W_{h}}\|p-v\|_{W} \leq C h^{r}\left(\|\vec{w}\|_{1+r}+\|p\|_{r}\right)
$$

From Section 2, we know that (4.6)-(4.7) and (4.8)-(4.9) have the following equivalent operator forms, respectively,

$$
\begin{aligned}
\lambda T \vec{u}=\vec{u}, & \sigma=S(\lambda \vec{u}), \\
\lambda_{h} T_{h} \vec{u}_{h}=\vec{u}_{h}, & \sigma_{h}=S_{h}\left(\lambda_{h} \vec{u}_{h}\right) .
\end{aligned}
$$

Moreover, $T$ and $T_{h}$ are all self-adjoint compact operators. 
Theorem 4.1. Assume that discrete inf-sup condition, (4.17) and (4.18) hold; let H be properly small; and $\left(\lambda^{h}, \vec{u}^{h}, \sigma^{h}\right)$ an approximate eigenpair obtained by Scheme 1 . Then there exists $\vec{u} \in M(\lambda)$ such that

$$
\begin{gathered}
\left\|\vec{u}^{h}-\vec{u}\right\|_{a} \leq C\left(H^{4 r}+h^{r}\right), \\
\left|\lambda^{h}-\lambda\right| \leq C\left(H^{8 r}+h^{2 r}\right) .
\end{gathered}
$$

Proof. From Brezzi-Babuska Theorem, (4.17), and (4.18), we deduce

$$
\left\|T \vec{f}-T_{h} \vec{f}\right\|_{V}+\left\|S \vec{f}-S_{h} \vec{f}\right\|_{W} \leq C h^{r}\|\vec{f}\|_{D^{\prime}} \quad \forall \vec{f} \in D .
$$

By virtue of Nitsche technique (see [29]) and (4.17), we derive

$$
\begin{aligned}
\left\|T \vec{f}-T_{h} \vec{f}\right\|_{D} & \leq C h^{r}\left(\inf _{q \in V_{h}}\|T \vec{f}-q\|_{V}+\inf _{v \in W_{h}}\|S \vec{f}-v\|_{W}\right) \\
& \leq C h^{2 r}\|\vec{f}\|_{D^{\prime}} \quad \forall f \in D .
\end{aligned}
$$

Using (4.24), we have

$$
\left\|T-T_{h}\right\|_{D}=\sup _{\vec{f} \in D,\|\vec{f}\|_{D}=1}\left\|T \vec{f}-T_{h} \vec{f}\right\|_{D} \leq C h^{2 r} \longrightarrow 0 \quad(h \longrightarrow 0)
$$

Hence, the conditions in Lemma 2.2 and Theorem 3.3 hold.

From (2.23), we know that

$$
\left|\lambda_{h}-\lambda\right|+\left\|\vec{u}_{h}-\vec{u}\right\|_{D} \leq C\left\|\left.\left(T-T_{h}\right)\right|_{M(\lambda)}\right\|_{D} \leq C h^{2 r}
$$

From (4.23), (4.18), and (4.17), we have

$$
\begin{gathered}
\left\|\left.\left(T-T_{h}\right)\right|_{M(\lambda)}\right\|_{a}=\sup _{\vec{u} \in M(\lambda), \vec{u} \neq 0} \frac{\left\|T \vec{u}-T_{h} \vec{u}\right\|_{a}}{\|\vec{u}\|_{a}} \leq C h^{r}, \\
\inf _{v \in W_{h}}\|\sigma-v\|_{W} \leq C h^{r}\|\sigma\|_{r} \leq C h^{r}\|\vec{u}\|_{D} .
\end{gathered}
$$

Substituting (4.26) and (4.27) into (3.7), we obtain (4.21). Substituting (4.21) and (4.28) into (3.8), we obtain (4.22).

\subsection{Mini Mixed Finite Element}

Consider two-scale discretization scheme of Mini mixed finite element for the Stokes eigenvalue problem (4.1)-(4.3) (Scheme 1). 
Mini element was established by Arnold et al. in 1984 (see [31]). Let $K^{h}$ be a regular triangulation of $\Omega$ under the meaning of paper [32], and

$$
S^{h}=\left\{v \in C(\bar{\Omega}):\left.v\right|_{\kappa} \in P_{1}, \kappa \in K^{h}\right\}, \quad S_{0}^{h}=S^{h} \bigcap H_{0}^{1}(\Omega)
$$

For any $\kappa \in K^{h}$, let $N_{1}, N_{2}$, and $N_{3}$ denote barycentric coordinates. Denote $B_{h}=\left\{v:\left.v\right|_{\kappa} \in\right.$ $\left.\operatorname{span}\left\{N_{1} N_{2} N_{3}\right\}, \kappa \in K^{h}\right\}$, and set

$$
V_{h}=\left(S_{0}^{h} \bigoplus B_{h}\right)^{2}, \quad W_{h}=S^{h} \bigcap L_{0}^{2}(\Omega)
$$

From [31], we know that Mini element satisfies discrete inf-sup condition. From the interpolation theory in Sobolev space, we conclude that (4.18) is valid. Hence, for $r$ satisfying (4.17), Scheme 1 for Mini mixed finite element is effective. Theorem 4.1 is valid.

\section{2. $P_{1}-P_{1}$ Mixed Finite Element}

Consider two-scale discretization scheme of $P_{1}-P_{1}$ mixed finite element for the Stokes eigenvalue problem (4.1)-(4.3) (Scheme 1).

Let $K^{2 h}$ be a regular triangulation of $\Omega$, and $K^{h}$ is the product of refining $K^{2 h}$ in the middle point. The $P_{1}-P_{1}$ mixed finite element space is defined by

$$
V_{h}=\left[S^{h} \bigcap H_{0}^{1}(\Omega)\right]^{2}, \quad W_{h}=S^{2 h} \bigcap L_{0}^{2}(\Omega)
$$

where $S^{2 h}$ and $S^{h}$ are piecewise continuous linear polynomial spaces defined on $K^{2 h}$ and $K^{h}$, respectively.

From [33, Proposition 3.3], we know that $P_{1}-P_{1}$ element satisfies discrete inf-sup condition. By the interpolation theory in Sobolev space, we conclude that (4.18) holds. Therefore, for $r$ satisfying (4.17), Scheme 1 for $P_{1}-P_{1}$ mixed finite element is effective. Theorem 4.1 is valid.

\section{Two-Scale Discretization Scheme for Eigenvalue Problem of Electric Field}

Consider the eigenvalue problem of electric field:

$$
\begin{gathered}
c^{2} \text { curl curl } \vec{u}=\omega^{2} \vec{u}, \quad \text { in } \Omega, \\
\operatorname{div} \vec{u}=0, \quad \text { in } \Omega, \\
\vec{u} \times \vec{\gamma}=0, \quad \text { on } \in \partial \Omega,
\end{gathered}
$$

where $\Omega$ is a polyhedron in $\mathbf{R}^{3}$ and $\vec{\gamma}$ is the outward normal unit vector on $\partial \Omega$. 
In physics, $\vec{u}$ in the above eigenvalue problem of electric field denotes electric field, $\omega$ denotes the time frequency, and $c$ is the speed of the light. Usually we set $\lambda=\omega^{2} / c^{2}$ which is called eigenvalue.

The spaces $H(\operatorname{curl}, \Omega), H_{0}(\operatorname{curl}, \Omega)$ are defined in the usual way:

$$
\begin{aligned}
& H(\operatorname{curl}, \Omega)=\left\{\vec{q} \in L_{2}(\Omega)^{3}: \operatorname{curl} \vec{q} \in L_{2}(\Omega)^{3}\right\} \\
& H_{0}(\operatorname{curl}, \Omega)=\left\{\vec{q} \in H(\operatorname{curl}, \Omega): \vec{q} \times\left.\vec{\gamma}\right|_{\partial \Omega}=0\right\}
\end{aligned}
$$

When $\Omega$ is convex polyhedron, we define the following function space:

$$
x=\left\{\vec{q} \in H_{0}(\operatorname{curl}, \Omega): \operatorname{div} \vec{q} \in L^{2}(\Omega)\right\} .
$$

Denote

$$
\begin{gathered}
(\vec{q}, \vec{\psi})_{0}=\int_{\Omega} \vec{q} \cdot \vec{\psi} d x, \quad\|\vec{q}\|_{0}=(\vec{q}, \vec{q})_{0}^{1 / 2} . \\
(\vec{q}, \vec{\psi})_{X}=(\operatorname{curl} \vec{q}, \operatorname{curl} \vec{\psi})_{0}+(\operatorname{div} \vec{q}, \operatorname{div} \vec{\psi})_{0^{\prime}} \quad\|\vec{q}\|_{X}=(\vec{q}, \vec{q})_{x}^{1 / 2} .
\end{gathered}
$$

From $[34,35]$, we see that $x \subset H^{1}(\Omega)^{3} ;(\vec{q}, \vec{\psi})_{X}$ is a coercive bilinear form in $X$, and $\|\vec{q}\|_{X}$ is a norm.

On the other hand, when $\Omega$ is nonconvex the maximal interior angle belongs to $(\pi, 2 \pi)$. In this situation the problem is relatively complicated. Let $E$ denote a set of reentrant edge with dihedral angles belonging to $(\pi, 2 \pi)$, and let $d$ denote the distance to the set $E: d(x)=$ $\operatorname{dist}\left(x, \cup_{e \in E} \bar{e}\right)$. We introduce a weight function $\omega_{r}$ which is a nonnegative smooth function with respect to $x$. It can be represented by $d^{r}$ in reentrant edge and angular domain. We write $\omega_{r} \simeq d^{r}$. Define the weight function space:

$$
\begin{aligned}
& L_{r}^{2}(\Omega)=\left\{v \in L_{\mathrm{loc}}^{2}(\Omega): \omega_{r} v \in L_{2}(\Omega)\right\} \\
& X_{r}=\left\{\vec{q} \in H_{0}(\operatorname{curl}, \Omega): \operatorname{div} \vec{q} \in L_{r}^{2}(\Omega)\right\} .
\end{aligned}
$$

Denote

$$
\begin{gathered}
(\vec{q}, \vec{\psi})_{L_{r}^{2}}=\int_{\Omega} \omega_{r}^{2} \vec{q} \cdot \vec{\psi} d x, \quad\|\vec{q}\|_{L_{r}^{2}}=(\vec{q}, \vec{q})_{L_{r}^{2}}^{1 / 2}, \\
(\vec{q}, \vec{\psi})_{X_{r}}=(\operatorname{curl} \vec{q}, \operatorname{curl} \vec{\psi})_{0}+(\operatorname{div} \vec{q}, \operatorname{div} \vec{\psi})_{L_{r}^{2},}\|\vec{q}\|_{X_{r}}=(\vec{q}, \vec{q})_{X_{r}}^{1 / 2} .
\end{gathered}
$$


Let $\sigma_{\Delta}^{N}$ be the following smallest singular exponent in the Laplace problem with homogenous Dirichlet boundary condition:

$$
\begin{gathered}
\left\{\phi \in H_{0}^{1}(\Omega): \Delta \phi \in L_{2}(\Omega)\right\} \subset \bigcap_{s<\sigma_{\Delta}^{D}} H^{s}(\Omega), \\
\left\{\phi \in H_{0}^{1}(\Omega): \Delta \phi \in L_{2}(\Omega)\right\} \not \subset H^{\sigma_{\Delta}^{D}}(\Omega) .
\end{gathered}
$$

From the regularity estimate we know $\sigma_{\Delta}^{D} \in(3 / 2,2)$. Let $r_{\min }=2-\sigma_{\Delta}^{D}$.

From [36, 37], we know that, for all $r \in\left(r_{\min }, 1\right)$, the seminorm $|\vec{q}|_{X_{r}}$ is a norm in $\chi_{r}$, and $\chi_{r} \cap H^{1}(\Omega)^{3}$ is dense in $\chi_{r}$.

In the following discussion, we will use $x_{r}, L_{r}^{2}(\Omega)$ both for non-convex and convex domain. We take $r \in\left(r_{\min }, 1\right)$ for non-convex domain; otherwise, we take $\chi_{r}=\chi, L_{r}^{2}(\Omega)=$ $L^{2}(\Omega)$.

By introducing Lagrange multiplier $\sigma,[36,38,39]$ changed (5.1)-(5.3) into the mixed variational formulation: find $(\lambda, \vec{u}, \sigma) \in \mathbf{R}^{+} \times X_{r} \times L_{r}^{2}(\Omega)$ such that

$$
\begin{gathered}
(\vec{u}, \vec{\psi})_{X_{r}}+(\operatorname{div} \vec{\psi}, \sigma)_{L_{r}^{2}}=\lambda(\vec{u}, \vec{\psi})_{0}, \quad \forall \vec{\psi} \in X_{r} \\
(\operatorname{div} \vec{u}, v)_{L_{r}^{2}}=0, \quad \forall v \in L_{r}^{2}(\Omega) .
\end{gathered}
$$

Let $K^{h}$ be a regular simplex partition (tetrahedral partition) of $\Omega$ with the mesh diameter $h$. Define the $P_{k+1}-P_{k}$ finite element space as follows:

$$
\begin{gathered}
V_{h}=\left\{\vec{q} \in C^{0}(\bar{\Omega})^{3}: \vec{q} \times\left.\vec{\gamma}\right|_{\partial \Omega}=0,\left.\vec{q}\right|_{\kappa} \in P_{k+1}(\kappa)^{3}, \forall \kappa \in K^{h}\right\}, \\
W_{h}=\left\{v \in C^{0}(\bar{\Omega}):\left.v\right|_{\kappa} \in P_{k}(\mathcal{\kappa}), \forall \mathcal{K} \in K^{h},\left.v\right|_{E_{h}}=0\right\} .
\end{gathered}
$$

Here we set $E_{h}=\left.\cup_{\kappa \in K^{h}, \partial \kappa \cap E \neq \phi} \kappa \cdot v\right|_{E_{h}}=0$ means that $v$ is zero on the tetrahedron where reentrant edge and angular point are adjacent.

Restricting (5.10)-(5.11) to the previous finite element space, we get discrete mixed variational form: find $\left(\lambda_{h}, \vec{u}_{h}, \sigma_{h}\right) \in \mathbf{R}^{+} \times \chi_{h} \times M_{h}$ such that

$$
\begin{gathered}
\left(\vec{u}_{h}, \vec{\psi}\right)_{X_{r}}+\left(\operatorname{div} \vec{\psi}, \sigma_{h}\right)_{L_{r}^{2}}=\lambda_{h}\left(\vec{u}_{h}, \vec{\psi}\right)_{0}, \quad \forall \vec{\psi} \in X_{h}, \\
\left(\operatorname{div} \vec{u}_{h}, v\right)_{L_{r}^{2}}=0, \quad \forall v \in M_{h} .
\end{gathered}
$$

Set

$$
\begin{gathered}
V=x_{r}, \quad\|\cdot\|_{V}=\|\cdot\|_{X_{r}} \\
W=L_{r}^{2}(\Omega), \quad\|\cdot\|_{W}=\|\cdot\|_{L_{r}^{2}} \\
D=L_{2}(\Omega)^{3}, \quad\|\cdot\|_{D}=\|\cdot\|_{0} \\
a(\vec{q}, \vec{\psi})=(\vec{q}, \vec{\psi})_{X_{r}{ }^{\prime}} \quad b(\vec{\psi}, v)=(\operatorname{div} \vec{\psi}, v)_{L_{r}^{2}} .
\end{gathered}
$$


Then (5.10)-(5.11) and (5.13)-(5.14) can be written in the forms of (2.3)-(2.4) and (2.5)-(2.6), respectively (we need to add $\rightarrow$ for the vector function, e.g., $u, \psi$ should be written in the forms of $\vec{u}, \vec{\psi})$.

We apply Scheme 1 to the eigenvalue problem of electric field (5.10)-(5.11). Adding the symbol $\rightarrow$ for the vector function we get two-scale discretization scheme of mixed finite element for solving the eigenvalue problem of electric field (5.10)-(5.11) which is still called Scheme 1.

It is easy to know that $a(\cdot, \cdot)$ and $b(\cdot, \cdot)$ are continuous bilinear forms on $V \times V$ and $V \times W$, respectively. $V$ is compact embedded in $D$ (when $\Omega$ is convex, it is valid obviously; when $\Omega$ is non-convex, see [36]).

Consider the source problem corresponding to (5.10)-(5.11).

Find $(\vec{w}, p) \in X_{r} \times L_{r}^{2}(\Omega)$ such that

$$
\begin{gathered}
(\vec{w}, \vec{\psi})_{X_{r}}+(\operatorname{div} \vec{\psi}, p)_{L_{r}^{2}}=(\vec{f}, \vec{\psi})_{0^{\prime}} \quad \forall \vec{\psi} \in X_{r}, \\
(\operatorname{div} \vec{w}, v)_{L_{r}^{2}}=0, \quad \forall v \in L_{r}^{2}(\Omega) .
\end{gathered}
$$

For the problem (5.16)-(5.17) and its $P_{k+1}-P_{k}$ element approximation, people have already proved the conditions in Brezzi-Babuska Theorem hold (see [38, 40,41]).

Therefore, we can define operators $T, S, T_{h}, S_{h}$; moreover, (5.10)-(5.11) and (5.13)(5.14) can be written in the forms of (2.23)-(2.24) and (2.25)-(2.26), respectively.

Lemma 5.1 is cited from the literature $[36,38]$.

Lemma 5.1. (5.1)-(5.3) is equivalent to (5.10)-(5.11), and the solutions of (5.10)-(5.11), $(\vec{u}, \sigma)$, satisfy $\sigma=S(\lambda \vec{u})=0$ and $\vec{u} \in X_{r}$ with $\operatorname{div} \vec{u}=0$.

Denote

$$
\varepsilon_{\lambda}(h)=\sup _{\vec{u} \in \widehat{M}(\lambda)} \inf _{\vec{\psi} \in V_{h}}\|T \vec{u}-\vec{\psi}\|_{a} .
$$

For the $P_{k+1}-P_{k}$ element approximation of (5.16)-(5.17), [38] proved that the condition of [24, Theorem 1] (i.e., [38, Theorem 4.3]) is valid, hence, there holds the following.

Lemma 5.2. For the $P_{k+1}-P_{k}$ element approximation of (5.16)-(5.17), there exists $r(h)>0, r(h) \rightarrow$ $0(h \rightarrow 0)$ such that

$$
\sup _{\vec{f} \in D, \vec{f} \neq 0} \frac{\left\|\left(T-T_{h}\right) \vec{f}\right\|_{V}}{\|\vec{f}\|_{D}} \leq r(h) .
$$

This lemma is very important. It tells us that $\left\|T-T_{h}\right\|_{D} \rightarrow 0(h \rightarrow 0)$. Based on this lemma, [38] also proved the following conclusion. 
Lemma 5.3. For the $P_{k+1}-P_{k}$ element approximation of (5.10)-(5.11), the following estimate is valid:

$$
\begin{gathered}
\left|\mathcal{\lambda}-\lambda_{h}\right| \leq C \varepsilon_{\lambda}(h)^{2}, \\
\left\|\vec{u}_{h}-\vec{u}\right\|_{a} \leq C \varepsilon_{\lambda}(h) .
\end{gathered}
$$

Theorem 5.4. Let $\left(\lambda^{h}, \vec{u}^{h}\right)$ be the approximate $P_{k+1}-P_{k}$ element eigenpair obtained by Scheme 1 ; then there exists $\vec{u} \in M(\lambda)$ such that

$$
\begin{gathered}
\left\|\vec{u}^{h}-\vec{u}\right\|_{a} \leq C\left(\varepsilon_{\lambda}(H)^{3}+\varepsilon_{\lambda}(h)\right), \\
\left|\lambda^{h}-\lambda\right| \leq C\left(\varepsilon_{\lambda}(H)^{6}+\varepsilon_{\lambda}(h)^{2}\right) .
\end{gathered}
$$

Proof. We use Theorem 3.3 to complete the proof. From (5.19) we see that the condition in Theorem 3.3 holds, and by Lemma 5.1 we know $\sigma=0$.

From (2.18), we deduce

$$
\begin{aligned}
\left\|\left.\left(T-T_{h}\right)\right|_{M(\lambda)}\right\|_{a} & =\sup _{\vec{u} \in \widehat{M}(\lambda)}\left\|T \vec{u}-T_{h} \vec{u}\right\|_{a} \\
& \leq C \sup _{\vec{u} \in \widehat{M}(\lambda)} \inf _{\vec{\psi} \in V_{h}}\|T \vec{u}-\vec{\psi}\|_{a} \\
& \leq C \varepsilon_{\lambda}(h) .
\end{aligned}
$$

From (5.20), we derive

$$
\left|\lambda_{H}-\lambda\right| \leq C \varepsilon_{\lambda}(H)^{2}
$$

Substituting (5.24) and (5.25) into (3.7), we get (5.22).

Since $\sigma=0$, (3.8) can be simplified to

$$
\left|\lambda^{h}-\lambda\right| \leq C\left\|\vec{u}^{h}-\vec{u}\right\|_{a}^{2}
$$

Substituting (5.22) into (5.26), we obtain (5.23).

Let $\sigma_{\Delta}^{N}$ be the smallest singular exponent in the Laplace problem with homogenous Neumann boundary condition, then $\sigma_{\Delta}^{N} \in(3 / 2,2)$. Denote $\tau=\min \left(r-r_{\min }, \sigma_{\Delta}^{N}-1\right)$.

Corollary 5.5. Under the condition of Theorem 5.4 , if $\Omega$ is a convex polyhedron there holds

$$
\begin{gathered}
\left\|\vec{u}^{h}-\vec{u}\right\|_{a} \leq C\left(H^{3}+h\right), \\
\left|\lambda^{h}-\lambda\right| \leq C\left(H^{6}+h^{2}\right) ;
\end{gathered}
$$


if $\Omega$ is a non-convex polyhedron, then

$$
\begin{aligned}
& \left\|\vec{u}^{h}-\vec{u}\right\|_{a} \leq C\left(H^{3 \mu}+h^{\mu}\right), \quad \forall \mu \in(0, \tau), \\
& \left|\lambda^{h}-\lambda\right| \leq C\left(H^{6 \mu}+h^{2 \mu}\right), \quad \forall \mu \in(0, \tau) .
\end{aligned}
$$

Proof. When $\Omega$ is a convex polyhedron, for any $\vec{u} \in M(\lambda)$ we have $\vec{u}=T(\lambda \vec{u}) \in H^{2}(\Omega)$ (see [35], or [42, equation (44)]); therefore

$$
\varepsilon_{\lambda}(H) \leq C H, \quad \varepsilon_{\lambda}(h) \leq C h .
$$

Substituting these two formulae into (5.22) and (5.23), we obtain (5.27) and (5.28), respectively. When $\Omega$ is non-convex, for all $\vec{u} \in M(\lambda)$, from [38, equation (36)] we know that

$$
\varepsilon_{\lambda}(H) \leq C H^{\mu}, \quad \varepsilon_{\lambda}(h) \leq C h^{\mu} .
$$

Substituting the above two formulae into (5.22) and (5.23), we obtain (5.29) and (5.30), respectively.

\section{Numerical Experiments}

In the following two examples, let $\lambda_{1, H}, \lambda_{2, H}, \ldots, \lambda_{4, H}$ be the first four eigenvalues computed by using mixed finite method directly on coarse mesh $K^{H}$ and $\lambda_{1, h}, \lambda_{2, h}, \ldots, \lambda_{4, h}$ the first four eigenvalues computed by using mixed finite method directly on fine mesh $K^{h}$. Let $\lambda_{1}^{h}, \lambda_{2}^{h}, \ldots, \lambda_{4}^{h}$ denote the first four eigenvalues computed by Scheme 1 on the meshes $K^{H}$ and $K^{h}$.

Example 6.1. Consider the Stokes eigenvalue problem (4.1)-(4.3), where $\Omega \subset \mathbf{R}^{2}$ is a unit square domain. The smallest eigenvalue $\lambda_{1}$ is approximately equal to 52.3446911 for this problem.

We adopt a uniform isosceles right triangulation for the domain $\Omega$ (the edge in each element is along three fixed directions), and we give an initial mesh in Figure 1 and refine the initial mesh in a uniform way (each triangle is divided into four congruent triangles) repeatedly to get meshes $K^{H}$ and $K^{h}$.

We solve this problem by Scheme 1 with Mini element. The numerical results are shown in Table 1.

From Table 1, we conclude that Scheme 1 can efficiently solve Stokes eigenvalue problem.

Example 6.2. Consider the eigenvalue problem of electric field (5.1)-(5.3), where $\Omega$ is a square domain $[0, \pi] \times[0, \pi]$ or an L-shaped domain $[-1,0] \times[-1,0] \cup[-1,1] \times[0,1]$. For the square domain, the first four exact eigenvalues are $\lambda_{1}=1, \lambda_{2}=1, \lambda_{3}=2, \lambda_{4}=4$ for this problem. For the L-shaped domain, the first four approximate eigenvalues are $\lambda_{1} \approx 1.475622, \lambda_{2} \approx$ $3.534031, \lambda_{3} \approx 9.869604, \lambda_{4} \approx 9.869604$. 
Abstract and Applied Analysis

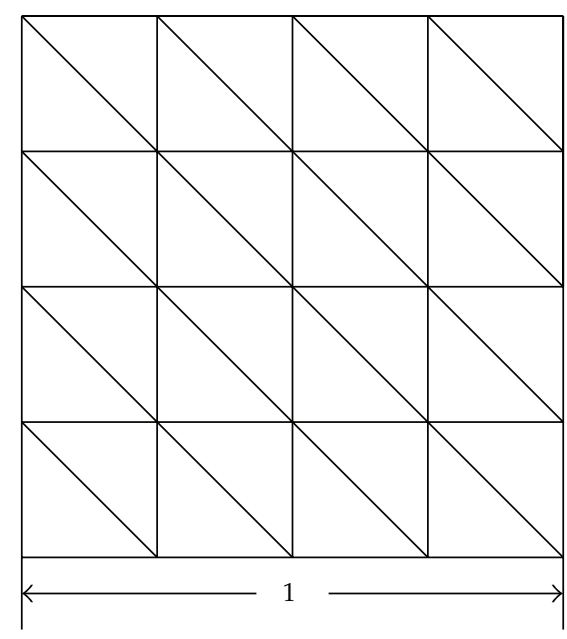

Figure 1

Table 1: The results on the square by Scheme 1 (Mini element) for Stokes eigenvalue problem.

\begin{tabular}{|c|c|c|c|c|c|}
\hline$k$ & $H$ & $h$ & $\lambda_{k, H}$ & $\lambda_{k, h}$ & $\lambda_{k}^{h}$ \\
\hline 1 & $\frac{\sqrt{2}}{8}$ & $\frac{\sqrt{2}}{64}$ & 57.50602342019 & 52.42255785241 & 52.42147553267 \\
\hline 1 & $\frac{\sqrt{2}}{10}$ & $\frac{\sqrt{2}}{100}$ & 55.61324143591 & 52.37622690147 & 52.37604993774 \\
\hline 1 & $\frac{\sqrt{2}}{12}$ & $\frac{\sqrt{2}}{144}$ & 54.59828590012 & 52.35983048690 & 52.35978975974 \\
\hline 2 & $\frac{\sqrt{2}}{8}$ & $\frac{\sqrt{2}}{64}$ & 103.5158846668 & 92.30714521284 & 92.29553836981 \\
\hline 2 & $\frac{\sqrt{2}}{10}$ & $\frac{\sqrt{2}}{100}$ & 99.36963738012 & 92.19619098643 & 92.19429134425 \\
\hline 2 & $\frac{\sqrt{2}}{12}$ & $\frac{\sqrt{2}}{144}$ & 97.12904494102 & 92.15848299868 & 92.15804753694 \\
\hline 3 & $\frac{\sqrt{2}}{8}$ & $\frac{\sqrt{2}}{64}$ & 110.7048253409 & 92.43050152401 & 92.39531304531 \\
\hline 3 & $\frac{\sqrt{2}}{10}$ & $\frac{\sqrt{2}}{100}$ & 103.8058661064 & 92.24032470162 & 92.23510195554 \\
\hline 3 & $\frac{\sqrt{2}}{12}$ & $\frac{\sqrt{2}}{144}$ & 100.1402638604 & 92.17887213187 & 92.17771885852 \\
\hline 4 & $\frac{\sqrt{2}}{8}$ & $\frac{\sqrt{2}}{64}$ & 159.7476076282 & 128.8864896622 & 128.7085892117 \\
\hline 4 & $\frac{\sqrt{2}}{10}$ & $\frac{\sqrt{2}}{100}$ & 148.7986378758 & 128.4767873556 & 128.4133574197 \\
\hline 4 & $\frac{\sqrt{2}}{12}$ & $\frac{\sqrt{2}}{144}$ & 142.5976379771 & 128.3089668708 & 128.3076834410 \\
\hline
\end{tabular}


Table 2: The results on the square by Scheme $1\left(P_{2}-P_{1}\right.$ element) for eigenvalue problem of electric field $(r=0)$.

\begin{tabular}{lccccc}
\hline$k$ & $H$ & $h$ & $\lambda_{k, H}$ & $\lambda_{k, h}$ & $\lambda_{k}^{h}$ \\
\hline 1 & $\frac{\sqrt{2}}{4}$ & $\frac{\sqrt{2}}{72}$ & 1.000489112 & 1.000000005 & 1.000000005 \\
1 & $\frac{\sqrt{2}}{6}$ & $\frac{\sqrt{2}}{72}$ & 1.000099609 & 1.000000005 & 1.000000005 \\
1 & $\frac{\sqrt{2}}{8}$ & $\frac{\sqrt{2}}{72}$ & 1.000031944 & 1.000000005 & 1.000000005 \\
2 & $\frac{\sqrt{2}}{4}$ & $\frac{\sqrt{2}}{72}$ & 1.000490210 & 1.000000005 & 1.000000005 \\
2 & $\frac{\sqrt{2}}{6}$ & $\frac{\sqrt{2}}{72}$ & 1.000099685 & 1.000000005 & 1.000000005 \\
2 & $\frac{\sqrt{2}}{8}$ & $\frac{\sqrt{2}}{72}$ & 1.000031956 & 1.0000000005 & 1.000000005 \\
3 & $\frac{\sqrt{2}}{4}$ & $\frac{\sqrt{2}}{72}$ & 2.006497695 & 2.0000000070 & 2.000000070 \\
3 & $\frac{\sqrt{2}}{6}$ & $\frac{\sqrt{2}}{72}$ & 2.001365268 & 2.000000070 & 2.000000070 \\
3 & $\frac{\sqrt{2}}{8}$ & $\frac{\sqrt{2}}{72}$ & 2.000442889 & 2.000000070 & 2.000000070 \\
4 & $\frac{\sqrt{2}}{4}$ & $\frac{\sqrt{2}}{72}$ & 4.028293524 & 4.000000321 & 4.000000333 \\
4 & $\frac{\sqrt{2}}{6}$ & $\frac{\sqrt{2}}{72}$ & 4.006078511 & 4.000000321 & 4.000000321 \\
4 & $\frac{\sqrt{2}}{8}$ & $\frac{\sqrt{2}}{72}$ & 4.001988912 & 4.0000000321 & 4.000000321 \\
\hline
\end{tabular}

We adopt a uniform isosceles right triangulation for $\Omega$ (the edge in each element is along three fixed directions, see Figure 2 for the L-shaped domain, and see Figure 1 for the square domain $[0, \pi] \times[0, \pi])$ to produce the meshes $K^{H}$ and $K^{h}$.

We use $P_{2}-P_{1}$ mixed finite element to solve this problem. The definition of $P_{2}-P_{1}$ mixed finite element space is given by

$$
\begin{gathered}
V_{h}=\left\{\vec{q} \in C^{0}(\bar{\Omega})^{2}: \vec{q} \times\left.\vec{\gamma}\right|_{\partial \Omega}=0,\left.\vec{q}\right|_{\kappa} \in P_{2}(\kappa)^{2}, \forall \kappa \in K^{h}\right\}, \\
W_{h}=\left\{v \in C^{0}(\bar{\Omega}):\left.v\right|_{\kappa} \in P_{1}(\kappa), \forall \kappa \in K^{h},\left.v\right|_{E_{h}}=0\right\} .
\end{gathered}
$$

We compute the first four approximate eigenvalues by using Scheme 1 with $P_{2}-P_{1}$ element on the mesh $K^{H}$ and $K^{h}$. The numerical results are listed in Tables 2, 3, and 4.

From Tables $2-4$, we conclude that Scheme 1 can efficiently solve the eigenvalue problem of electric field. 


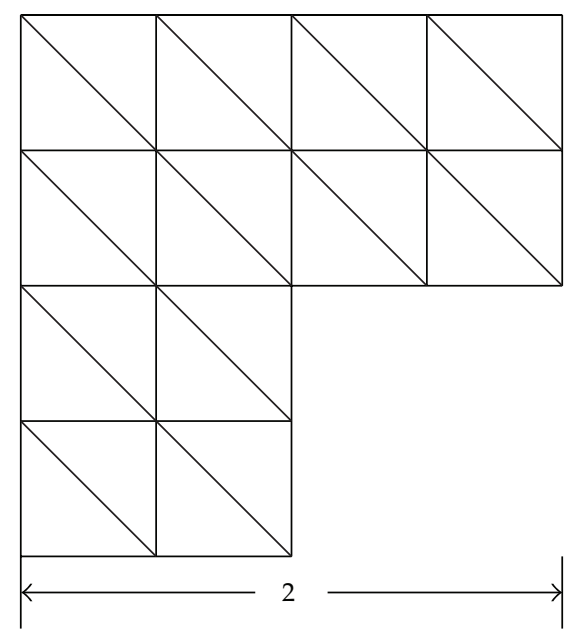

Figure 2

Table 3: The results on the L-shaped domain by Scheme $1\left(P_{2}-P_{1}\right.$ element) for eigenvalue problem of electric field $(r=0.5)$.

\begin{tabular}{lllccc}
\hline$k$ & $H$ & $h$ & $\lambda_{k, H}$ & $\lambda_{k, h}$ & $\lambda_{k}^{h}$ \\
\hline 1 & $\frac{\sqrt{2}}{4}$ & $\frac{\sqrt{2}}{72}$ & 3.004954998 & 2.557217952 & 2.557241250 \\
1 & $\frac{\sqrt{2}}{6}$ & $\frac{\sqrt{2}}{72}$ & 2.896205625 & 2.557217952 & 2.557225625 \\
1 & $\frac{\sqrt{2}}{8}$ & $\frac{\sqrt{2}}{72}$ & 2.842087274 & 2.557217952 & 2.557221723 \\
2 & $\frac{\sqrt{2}}{4}$ & $\frac{\sqrt{2}}{72}$ & 3.686002173 & 3.536967001 & 3.536967768 \\
2 & $\frac{\sqrt{2}}{6}$ & $\frac{\sqrt{2}}{72}$ & 3.623341929 & 3.536967001 & 3.536967079 \\
2 & $\frac{\sqrt{2}}{8}$ & $\frac{\sqrt{2}}{72}$ & 3.595097794 & 3.536967001 & 3.536967017 \\
3 & $\frac{\sqrt{2}}{4}$ & $\frac{\sqrt{2}}{72}$ & 9.938579092 & 9.869605192 & 9.869605219 \\
3 & $\frac{\sqrt{2}}{6}$ & $\frac{\sqrt{2}}{72}$ & 9.884458574 & 9.869605192 & 9.869605192 \\
3 & $\frac{\sqrt{2}}{8}$ & $\frac{\sqrt{2}}{72}$ & 9.874470046 & 9.869605192 & 9.869605192 \\
4 & $\frac{\sqrt{2}}{4}$ & $\frac{\sqrt{2}}{72}$ & 9.938761731 & 9.869605192 & 9.869605221 \\
4 & $\frac{\sqrt{2}}{6}$ & $\frac{\sqrt{2}}{72}$ & 9.884463697 & 9.869605192 & 9.869605192 \\
4 & $\frac{\sqrt{2}}{8}$ & $\frac{\sqrt{2}}{72}$ & 9.874472304 & 9.869605192 & 9.869605192 \\
\hline
\end{tabular}


Table 4: The results on the L-shaped domain by Scheme $1\left(P_{2}-P_{1}\right.$ element) for eigenvalue problem of electric field $(r=0.95)$.

\begin{tabular}{lllccc}
\hline$k$ & $H$ & $h$ & $\lambda_{k, H}$ & $\lambda_{k, h}$ & $\lambda_{k}^{h}$ \\
\hline 1 & $\frac{\sqrt{2}}{4}$ & $\frac{\sqrt{2}}{72}$ & 1.913411786 & 1.517146260 & 1.517159275 \\
1 & $\frac{\sqrt{2}}{6}$ & $\frac{\sqrt{2}}{72}$ & 1.779172346 & 1.517146260 & 1.517148955 \\
1 & $\frac{\sqrt{2}}{8}$ & $\frac{\sqrt{2}}{72}$ & 1.723917701 & 1.517146260 & 1.517147418 \\
2 & $\frac{\sqrt{2}}{4}$ & $\frac{\sqrt{2}}{72}$ & 3.589241343 & 3.534187594 & 3.534187611 \\
2 & $\frac{\sqrt{2}}{6}$ & $\frac{\sqrt{2}}{72}$ & 3.558323120 & 3.534187594 & 3.534187595 \\
2 & $\frac{\sqrt{2}}{8}$ & $\frac{\sqrt{2}}{72}$ & 3.547766251 & 3.534187594 & 3.534187595 \\
3 & $\frac{\sqrt{2}}{4}$ & $\frac{\sqrt{2}}{72}$ & 9.937421407 & 9.869605190 & 9.869605213 \\
3 & $\frac{\sqrt{2}}{6}$ & $\frac{\sqrt{2}}{72}$ & 9.884270121 & 9.869605190 & 9.869605191 \\
3 & $\frac{\sqrt{2}}{8}$ & $\frac{\sqrt{2}}{72}$ & 9.874420606 & 9.869605190 & 9.869605191 \\
4 & $\frac{\sqrt{2}}{4}$ & $\frac{\sqrt{2}}{72}$ & 9.937916802 & 9.869605191 & 9.869605216 \\
4 & $\frac{\sqrt{2}}{6}$ & $\frac{\sqrt{2}}{72}$ & 9.884282799 & 9.869605191 & 9.869605190 \\
4 & $\frac{\sqrt{2}}{8}$ & $\frac{\sqrt{2}}{72}$ & 9.874424604 & 9.869605191 & 9.869605190 \\
\hline
\end{tabular}

In this paper, $C$ denotes a positive constant independent of $h$, which may stand for different values at its different occurrences.

\section{Acknowledgments}

This work is supported by the National Natural Science Foundation of China (no. 11161012) and Science and Technology Foundation of Guizhou Province of China (no. [2011]2111).

\section{References}

[1] J. Xu, "A new class of iterative methods for nonselfadjoint or indefinite problems," SIAM Journal on Numerical Analysis, vol. 29, no. 2, pp. 303-319, 1992.

[2] J. Xu, "A novel two-grid method for semilinear elliptic equations," SIAM Journal on Scientific Computing, vol. 15, no. 1, pp. 231-237, 1994.

[3] J. Xu, "Two-grid discretization techniques for linear and nonlinear PDEs," SIAM Journal on Numerical Analysis, vol. 33, no. 5, pp. 1759-1777, 1996.

[4] M. Cai, M. Mu, and J. Xu, "Numerical solution to a mixed Navier-Stokes/Darcy model by the twogrid approach," SIAM Journal on Numerical Analysis, vol. 47, no. 5, pp. 3325-3338, 2009. 
[5] Y. He, J. Xu, A. Zhou, and J. Li, "Local and parallel finite element algorithms for the Stokes problem," Numerische Mathematik, vol. 109, no. 3, pp. 415-434, 2008.

[6] J. Li, "Investigations on two kinds of two-level stabilized finite element methods for the stationary Navier-Stokes equations," Applied Mathematics and Computation, vol. 182, no. 2, pp. 1470-1481, 2006.

[7] M. Mu and J. Xu, "A two-grid method of a mixed Stokes-Darcy model for coupling fluid flow with porous media flow," SIAM Journal on Numerical Analysis, vol. 45, no. 5, pp. 1801-1813, 2007.

[8] C.-S. Chien and B.-W. Jeng, "A two-grid discretization scheme for semilinear elliptic eigenvalue problems," SIAM Journal on Scientific Computing, vol. 27, no. 4, pp. 1287-1340, 2006.

[9] J. Xu and A. Zhou, "A two-grid discretization scheme for eigenvalue problems," Mathematics of Computation, vol. 70, no. 233, pp. 17-25, 2001.

[10] Q. Lin and G. Q. Xie, "Acceleration of FEA for eigenvalue problems," Bulletin of Science, vol. 26, pp. 449-452, 1981.

[11] I. H. Sloan, "Iterated Galerkin method for eigenvalue problems," SIAM Journal on Numerical Analysis, vol. 13, no. 5, pp. 753-760, 1976.

[12] X. Dai and A. Zhou, "Three-scale finite element discretizations for quantum eigenvalue problems," SIAM Journal on Numerical Analysis, vol. 46, no. 1, pp. 295-324, 2007/08.

[13] X. Gong, L. Shen, D. Zhang, and A. Zhou, "Finite element approximations for Schrödinger equations with applications to electronic structure computations," Journal of Computational Mathematics, vol. 26, no. 3, pp. 310-323, 2008.

[14] H. Chen, F. Liu, and A. Zhou, "A two-scale higher-order finite element discretization for Schrödinger equation," Journal of Computational Mathematics, vol. 27, no. 2-3, pp. 315-337, 2009.

[15] K. Kolman, "A two-level method for nonsymmetric eigenvalue problems," Acta Mathematicae Applicatae Sinica, vol. 21, no. 1, pp. 1-12, 2005.

[16] Y. Yang and X. Fan, "Generalized Rayleigh quotient and finite element two-grid discretization schemes," Science in China A, vol. 52, no. 9, pp. 1955-1972, 2009.

[17] H. Bi and Y. Yang, "A two-grid method of the non-conforming Crouzeix-Raviart element for the Steklov eigenvalue problem," Applied Mathematics and Computation, vol. 217, no. 23, pp. 9669-9678, 2011.

[18] Q. Li and Y. Yang, "A two-grid discretization scheme for the Steklov eigenvalue problem," Journal of Applied Mathematics and Computing, vol. 36, no. 1-2, pp. 129-139, 2011.

[19] H. Chen, S. Jia, and H. Xie, "Postprocessing and higher order convergence for the mixed finite element approximations of the Stokes eigenvalue problems," Applications of Mathematics, vol. 54, no. 3, pp. 237-250, 2009.

[20] Y. D. Yang, "Iterated Galerkin method and Rayleigh quotient for accelerating convergence of eigenvalue problems," Chinese Journal of Engineering Mathematics, vol. 25, no. 3, pp. 480-488, 2008.

[21] Y. Yang and H. Bi, "Two-grid finite element discretization schemes based on shifted-inverse power method for elliptic eigenvalue problems," SIAM Journal on Numerical Analysis, vol. 49, no. 4, pp. 16021624, 2011.

[22] L. N. Trefethen and D. Bau,, Numerical Linear Algebra, SIAM, Philadelphia, Pa, USA, 1997.

[23] I. Babuška and J. Osborn, "Eigenvalue problems," in Finite Element Methods(Part 1), Handbook of Numerical Analysis, P. G. Ciarlet and J. L. Lions, Eds., pp. 641-787, Elsevier Science, North Holland, The Netherlands, 1991.

[24] D. Boffi, F. Brezzi, and L. Gastaldi, "On the convergence of eigenvalues for mixed formulations," Annali della Scuola Normale Superiore di Pisa. Classe di Scienze, vol. 25, no. 1-2, pp. 131-154, 1997.

[25] B. Mercier, J. Osborn, J. Rappaz, and P.-A. Raviart, "Eigenvalue approximation by mixed and hybrid methods," Mathematics of Computation, vol. 36, no. 154, pp. 427-453, 1981.

[26] V. Girault and P.-A. Raviart, Finite Element Methods for Navier-Stokes Equations, vol. 5 of Theory and Algorithms, Springer, Heidelberg, Germany, 1986.

[27] C. Bernardi and G. Raugel, "Analysis of some finite elements for the Stokes problem," Mathematics of Computation, vol. 44, no. 169, pp. 71-79, 1985.

[28] F. Brezzi and M. Fortin, Mixed and Hybrid Finite Element Methods, vol. 15, Springer, New York, NY, USA, 1991.

[29] R. Stenberg, "Analysis of mixed finite elements methods for the Stokes problem: a unified approach," Mathematics of Computation, vol. 42, no. 165, pp. 9-23, 1984.

[30] R. B. Kellogg and J. E. Osborn, "A regularity result for the Stokes problem in a convex polygon," Journal of Functional Analysis, vol. 21, no. 4, pp. 397-431, 1976.

[31] D. N. Arnold, F. Brezzi, and M. Fortin, "A stable finite element for the Stokes equations," Calcolo, vol. 21, no. 4, pp. 337-344, 1984. 
[32] P. G. Ciarlet, "Basic error estimates for elliptic problems," in Finite Element Methods(Part1), Handbook of Numerical Analysis, P. G. Ciarlet and J. L. Lions, Eds., vol. 3, pp. 21-343, Elsevier Science, North Holland, The Netherlands, 1991.

[33] R. Verfürth, "Error estimates for a mixed finite element approximation of the Stokes equations," RAIRO Analyse Numérique, vol. 18, no. 2, pp. 175-182, 1984.

[34] C. Amrouche, C. Bernardi, M. Dauge, and V. Girault, "Vector potentials in three-dimensional nonsmooth domains," Mathematical Methods in the Applied Sciences, vol. 21, no. 9, pp. 823-864, 1998.

[35] M. Costabel, "A coercive bilinear form for Maxwell's equations," Journal of Mathematical Analysis and Applications, vol. 157, no. 2, pp. 527-541, 1991.

[36] P. Ciarlet, Jr., "Augmented formulations for solving Maxwell equations," Computer Methods in Applied Mechanics and Engineering, vol. 194, no. 2-5, pp. 559-586, 2005.

[37] M. Costabel and M. Dauge, "Weighted regularization of Maxwell equations in polyhedral domains. A rehabilitation of nodal finite elements," Numerische Mathematik, vol. 93, no. 2, pp. 239-277, 2002.

[38] A. Buffa, P. Ciarlet, Jr., and E. Jamelot, "Solving electromagnetic eigenvalue problems in polyhedral domains with nodal finite elements," Numerische Mathematik, vol. 113, no. 4, pp. 497-518, 2009.

[39] F. Kikuchi, "Mixed and penalty formulations for finite element analysis of an eigenvalue problem in electromagnetism," Computer Methods in Applied Mechanics and Engineering, vol. 64, pp. 509-521, 1987.

[40] P. Ciarlet, Jr. and V. Girault, "inf-sup condition for the 3D, $P_{2}$-iso- $P_{1}$ Taylor-Hood finite element application to Maxwell equations," Comptes Rendus Mathématique, vol. 335, no. 10, pp. 827-832, 2002.

[41] P. Ciarlet, Jr. and G. Hechme, "Computing electromagnetic eigenmodes with continuous Galerkin approximations," Computer Methods in Applied Mechanics and Engineering, vol. 198, no. 2, pp. 358-365, 2008.

[42] D. Boffi, P. Fernandes, L. Gastaldi, and I. Perugia, "Computational models of electromagnetic resonators: analysis of edge element approximation," SIAM Journal on Numerical Analysis, vol. 36, no. 4, pp. 1264-1290, 1999. 


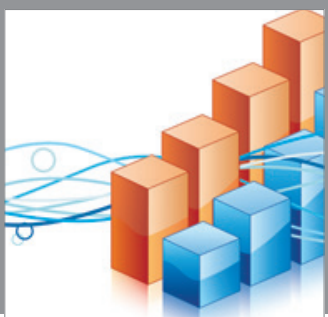

Advances in

Operations Research

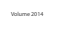

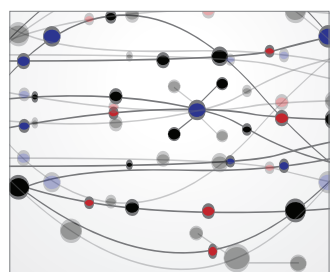

\section{The Scientific} World Journal
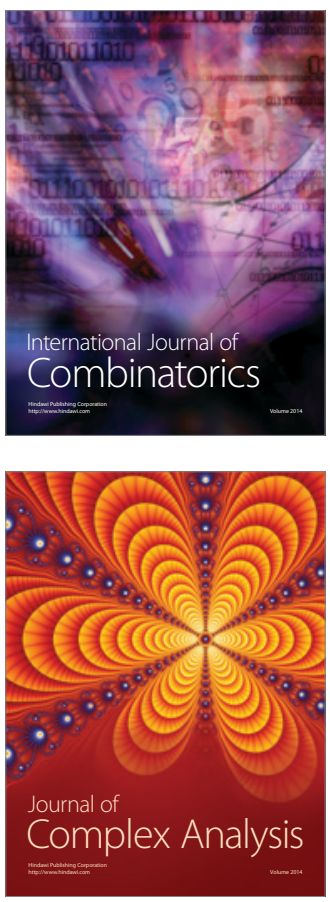

International Journal of

Mathematics and

Mathematical

Sciences
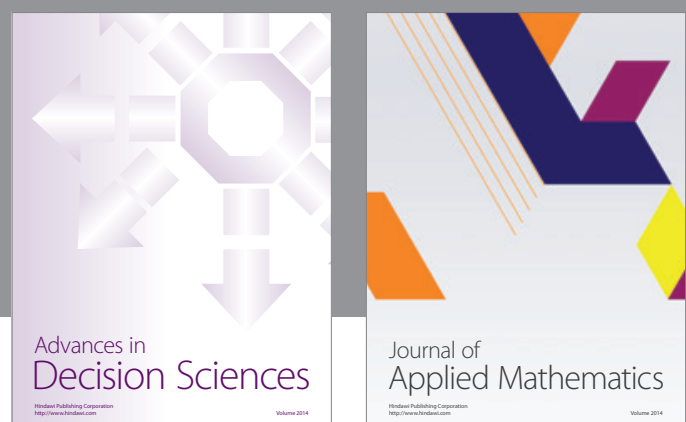

Journal of

Applied Mathematics
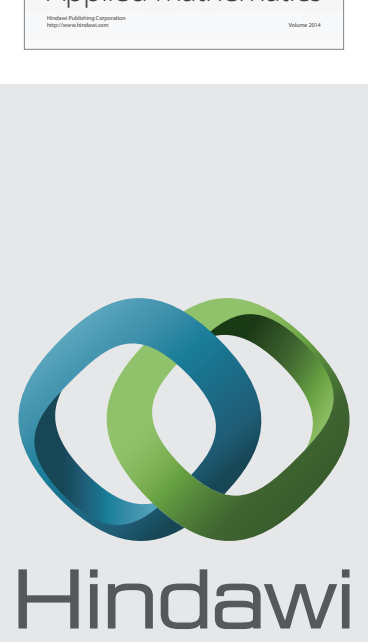

Submit your manuscripts at http://www.hindawi.com
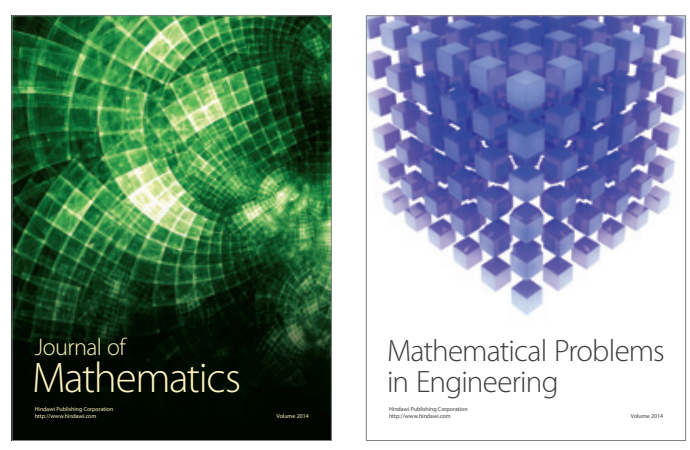

Mathematical Problems in Engineering
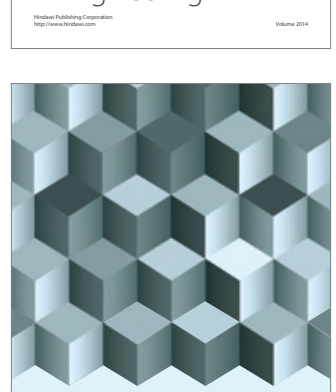

Journal of

Function Spaces
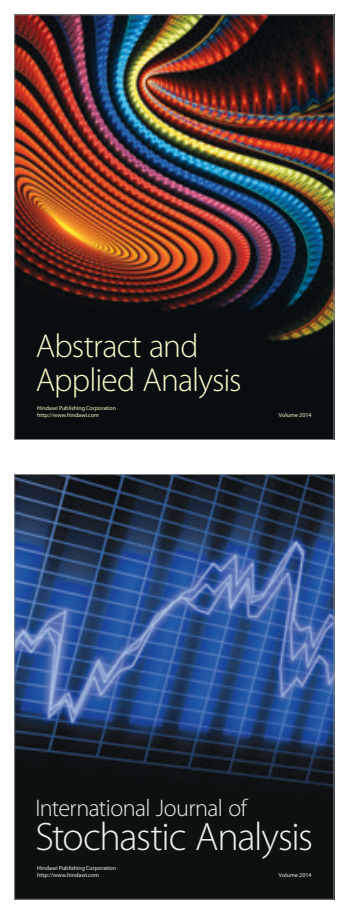

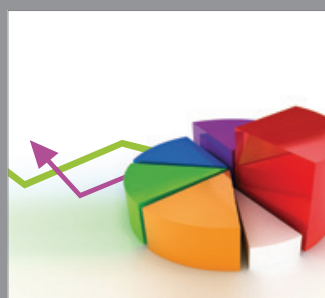

ournal of

Probability and Statistics

Promensencen
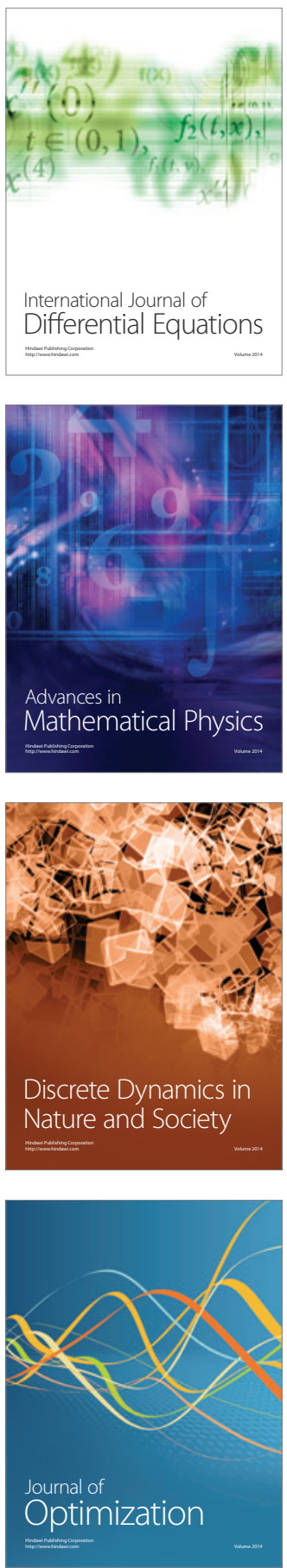